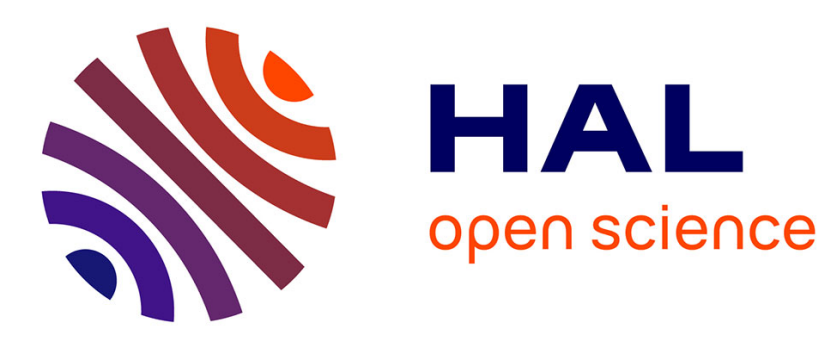

\title{
Dynamics of a thin liquid film interacting with an oscillating nano-probe
}

René Ledesma-Alonso, Philippe Tordjeman, Dominique Legendre

\section{To cite this version:}

René Ledesma-Alonso, Philippe Tordjeman, Dominique Legendre. Dynamics of a thin liquid film interacting with an oscillating nano-probe. Ethnopolitics, 2014, vol. 10 ( $\left.\mathrm{n}^{\circ} 39\right)$, pp. 7736-7752. $10.1039 / \mathrm{c} 4 \mathrm{sm} 01152 \mathrm{j}$. hal-01105390

\section{HAL Id: hal-01105390 \\ https://hal.science/hal-01105390}

Submitted on 20 Jan 2015

HAL is a multi-disciplinary open access archive for the deposit and dissemination of scientific research documents, whether they are published or not. The documents may come from teaching and research institutions in France or abroad, or from public or private research centers.
L'archive ouverte pluridisciplinaire HAL, est destinée au dépôt et à la diffusion de documents scientifiques de niveau recherche, publiés ou non, émanant des établissements d'enseignement et de recherche français ou étrangers, des laboratoires publics ou privés. 


\section{Open Archive TOULOUSE Archive Ouverte (OATAO)}

OATAO is an open access repository that collects the work of Toulouse researchers and makes it freely available over the web where possible.

This is an author-deposited version published in : http://oatao.univ-toulouse.fr/ Eprints ID : 12288

To link to this article : DOI:10.1039/c4sm01152j

http://dx.doi.org/10.1039/c4sm01152j

To cite this this version : Ledesma-Alonso, René and Tordjeman, Philippe and Legendre, Dominique Dynamics of a thin liquid film interacting with an oscillating nano-probe. (2014) Soft Matter, vol. 10 (n³9). pp. 7736-7752. ISSN 1744-683X

Any correspondance concerning this service should be sent to the repository administrator: staff-oatao@listes-diff.inp-toulouse.fr 


\title{
Dynamics of a thin liquid film interacting with an oscillating nano-probe
}

\begin{abstract}
René Ledesma-Alonso, ${ }^{*}$ Philippe Tordjeman and Dominique Legendre
The dynamic interaction between a local probe and a viscous liquid film, which provokes the deformation of the latter, has been studied. The pressure difference across the air-liquid interface is calculated with a modified Young-Laplace equation, which takes into account the effects of gravity, surface tension, and liquid film-substrate and probe-liquid attractive interaction potentials. This pressure difference is injected into the lubrication approximation equation, in order to depict the evolution of a viscous thinfilm. Additionally, a simple periodic function is added to an average separation distance, in order to define the probe motion. The aforementioned coupled equations, which describe the liquid film dynamics, were analysed and numerically solved. The liquid surface undergoes a periodic motion: the approaching probe provides an input energy to the film, which is stored by the latter by increasing its surface deformation; afterwards, when the probe moves away, an energy dissipation process occurs as the surface attempts to recover its original flat shape. Asymptotic regimes of the film surface oscillation are discerned, for extreme probe oscillation frequencies, and several length, wavenumber and time scales are yielded from our analysis, which is based on the Hankel transform. For a given probe-liquidsubstrate system, with well-known physical and geometric parameters, a periodic stationary regime and instantaneous and delayed probe wetting events are discerned from the numerical results, depending on the combination of oscillation parameters. Our results provide an interpretation of the probe-liquid film coupling phenomenon, which occurs whenever an AFM test is performed over a liquid sample.
\end{abstract}

\section{Introduction}

Over the past two decades, the application of dynamic mode atomic force microscopy (AFM) techniques has become a current practice for scanning soft matter samples. ${ }^{1}$ Nevertheless, the choice of AFM imaging mode should be made carefully in order to prevent inconveniences and undesired phenomena. Indeed, whenever a probe is brought into close proximity to a surface, molecules jump from the surface to the probe ${ }^{2}$ due to the attractive van der Waals tip sample interaction. This statement has been extensively discussed ${ }^{1,3,4}$ and observed experimentally, when a probe is brought close to a high temperature solid sample ${ }^{5,6}$ and to polymeric liquid films. ${ }^{7,8}$ As a probe quasistatically approaches a liquid sample, or a liquid layer deposited over a solid substrate, the liquid surface performs a jump towards the probe at a minimum separation distance. ${ }^{9-11}$ The intermittent contact mode (IC-AFM) reduces the probe-sample interaction time, by oscillating the probe in the vicinity of the sample surface and provoking a soft probe-sample contact. Images of liquid droplets using IC-AFM had been obtained, ${ }^{12,13}$ also registering a large phase contrast when the probe-liquid

Université de Toulouse, INPT-CNRS, Institut de Mécanique des Fluides de Toulouse (IMFT), 1 Allée du Professeur Camille Soula, 31400 Toulouse, France. E-mail: rledesma@imft.fr; Tel: +330534322802 contact occurs, which indicates the presence of an energy dissipation phenomenon. Indeed, every time the probe comes close to the liquid sample surface, a capillary neck forms between the probe and the sample. ${ }^{14,15}$ This may cause as well the liquid volume to split into two parts, one remaining as the sample and the other placed over the probe surface.

Imaging of the droplet profile using non-contact mode (NCAFM), which avoids the probe-liquid contact, has been shown as a possible solution..$^{1,16-22}$ A recipe to obtain good resolution topographies, consisting of the use of an oscillation frequency about $100 \mathrm{~Hz}$ higher than the cantilever resonance frequency, a free oscillation amplitude of around $10 \mathrm{~nm}$ and a probe-sample distance between 22 and $50 \mathrm{~nm}$, which is heuristically determined, has been recently exposed and successfully employed. ${ }^{20,21,23}$ If larger amplitudes or shorter probe-sample distances are used, an "accidental" contact between the tip and the sample is provoked and, as a consequence, distorted droplet profiles are captured. Although the proposed methodology provides quality results, a formal justification of this experimental combination of parameters still remains to be revealed.

Despite the non-intrusive nature of NC-AFM, the surface of a soft sample encounters the growth of a nano-protuberance under the action of the oscillating probe. ${ }^{24}$ Using a Kelvin-Voigt viscoelastic material to model the soft sample, in which inertial effects are disregarded, and a forced non-linear damped 
oscillator, including a sphere/flat surface interaction force, to mimic the NC-AFM operation, ${ }^{25}$ the sample deformation is estimated after the model parameters are determined by fitting experimental data. When NC-AFM experiments are performed, it is advisable to use stiff cantilevers, which provides cantilever deflection stability, and to minimize the probe-liquid separation distance, which increases the probe sensitivity, in order to accomplish true atomic resolution. In this scenario, the suitable scanning parameters must be defined to prevent the probe-soft sample contact and the loss of the original shape of the sample and shrinking of volume. Therefore, the NC-AFM jump-tocontact distance and the sample surface deformation should be deduced and compared to those for a static probe-liquid interaction.

In this paper, we present a theoretical and numerical study of the liquid film dynamics, generated by its interaction with an oscillating nano-probe. First, in Section II, we present equations that describe the probe and film dynamics. In Section III, we describe an implemented pseudo-spectral method to solve the probe-film dynamics. Section IV is devoted to the results of the numerical simulations, performed for different probe oscillation conditions. In Section V, we submit a theoretical analysis in the wavenumber domain and a solution for the film surface position. Section VI reports the critical oscillation parameters that lead to the probe wetting. Finally, in Section VII we discuss the consequences of the probe-liquid dynamic coupling on AFM experimental situations.

\section{Problem formulation}

A liquid film of thickness $E$, density $\rho$, dynamic viscosity $\mu$ and air-liquid surface tension $\gamma$ deposited over a flat horizontal substrate, as shown in Fig. 1, is considered. Within a cylindrical axisymmetric coordinate system, the position of the film free surface $z=\eta$ is a function of the radial position $r$ and time $t$. When the film surface is perturbed from its original flat shape

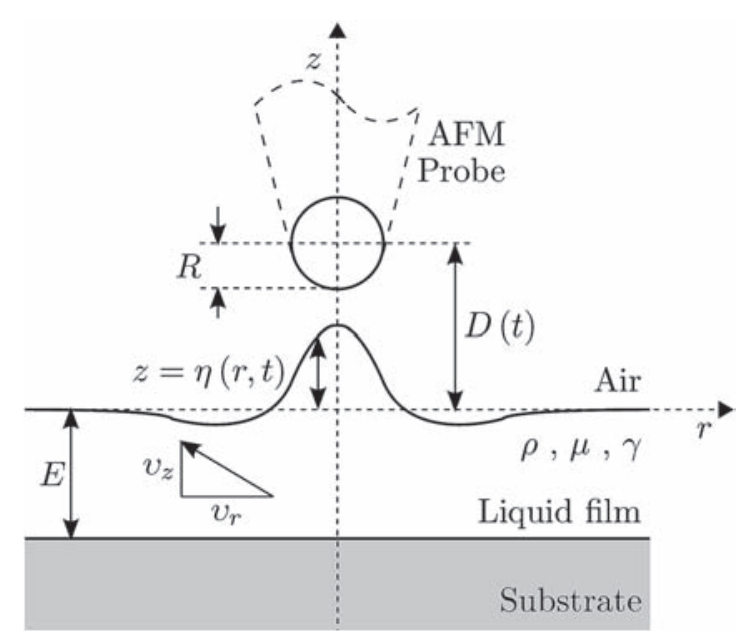

Fig. 1 Scheme of the liquid film and the deformation of its surface due to its interaction with a probe. An oscillating sphere has been used as an AFM probe model. The geometric and physical parameters are defined in the text. $\eta=0$ due to its interaction with an oscillating probe, a periodic response of the liquid is expected. Herein, the periodic probe motion is described by the following expression:

$$
D=\bar{D}+A \cos (\omega t),
$$

where $\bar{D}$ is the time-average probe position, $A$ is the oscillation amplitude and $\omega$ is the angular frequency.

In addition, in a liquid film of thickness below the corresponding capillary length, a viscous flow is also envisaged. Let us define $v_{r}$ and $v_{z}$ as the radial and axial components of the velocity field, respectively. The corresponding film boundary conditions, of no-slip at the substrate and shear-free at the free surface, ${ }^{26,27}$ are given by:

$$
\begin{aligned}
& v_{r}=0 \quad \text { at } z=-E, \\
& \frac{\partial v_{r}}{\partial z}=0 \quad \text { at } z=\eta .
\end{aligned}
$$

In addition, since the velocity field $\left(v_{r}, v_{z}\right)$ and the film free surface velocity in the direction normal to the surface should be equal, in order to respect the mass conservation, the kinematic condition at the liquid surface ${ }^{26,28}$ is written as:

$$
\frac{\partial \eta}{\partial t}=v_{z}-v_{r} \frac{\partial \eta}{\partial r} \quad \text { at } z=\eta
$$

and the pressure field $P$ within the liquid film is identified as the addition of the air atmospheric pressure $P_{0}$, which is considered to be constant, and the pressure difference $\Delta P$ across the interface, located at $z=\eta$. Therefore, for a thin viscous film, the momentum and continuity equations reduce to a typical Reynolds lubrication equation:

$$
\frac{\partial \eta}{\partial t}=\frac{1}{r} \frac{\partial}{\partial r}\left[r\left\{\frac{[E+\eta]^{3}}{3 \mu} \frac{\partial \Delta P}{\partial r}\right\}\right] .
$$

Solving eqn (4) for $\Delta P$ shows that this pressure difference also represents the effect of viscous drainage within the thin film, due to the surface motion.

According to the Hamaker theory, ${ }^{29}$ which takes into account the effect of van der Waals (vdW) forces to explain the interaction between macroscopic objects, the liquid film interacts with the surrounding bodies, including the substrate. In the present work, a film disturbance is created by the approach of a local probe, which, for simplicity, is considered to be a rigid sphere of radius $R .^{10}$ Disregarding the air density, the pressure difference across the interface is decomposed as:

$$
\Delta P=\rho g \eta+2 \gamma \kappa+\Pi_{1 \mathrm{~s}}+\Pi_{\mathrm{pl}}
$$

where $g$ is the acceleration of gravity, $\kappa$ is the local mean curvature, $\Pi_{\mathrm{ls}}$ and $\Pi_{\mathrm{pl}}$ are the liquid-substrate and the probeliquid interaction potentials. In the presented reference system, the local mean curvature takes the form:

$$
\kappa=-\frac{1}{2}\left\{\frac{1}{r} \frac{\partial}{\partial r}\left[r \frac{\partial \eta}{\partial r}\left\{\left[\frac{\partial \eta}{\partial r}\right]^{2}+1\right\}^{-1 / 2}\right]\right\} .
$$


Each interaction potential that contributes to the interface displacement corresponds to the potential energy difference between the perturbed state and the originally undisturbed state. The potential field created by the interaction between the substrate and the liquid film, at $z=\eta$, is described by:

$$
\Pi_{\mathrm{ls}}=-\frac{H_{\mathrm{ls}}}{6 \pi}\left\{\frac{1}{[E+\eta]^{3}}-\frac{1}{[E]^{3}}\right\},
$$

where $H_{\mathrm{ls}}$ is the Hamaker constant of the liquid-substrate interaction. In turn, a local probe placed at a distance $D$ from the film surface, as shown in Fig. 1, provokes the displacement of the originally flat interface. Thus, at $z=\eta$, the interaction potential mutually exerted between the spherical probe and the liquid film is given by:

$$
\Pi_{\mathrm{pl}}=-\frac{4 H_{\mathrm{pl}} R^{3}}{3 \pi} \frac{1}{\left\{[D-\eta]^{2}+r^{2}-R^{2}\right\}^{3}},
$$

where $H_{\mathrm{pl}}$ is the Hamaker constant of the probe-liquid interaction. The procedure to obtain eqn (7) and (8) has been previously detailed. ${ }^{11}$ The combination of eqn (4)-(8) describes the behaviour of the film surface in terms of the radial position and time, as well as the physical and geometric parameters. Note that any driving function $D(t)$ can be embedded into eqn (8), including the AFM-like periodic motion of the probe given in eqn (1).

Let us nondimensionalize using the probe radius $R$ and the average gap $\xi=\bar{D}-R$ as the characteristic radial and deformation length scales, respectively. Thus, we have:

$$
\begin{array}{lll}
E^{*}=E / R, & D^{*}=D / R, & r^{*}=r / R, \\
z^{*}=z / \xi, & \eta^{*}=\eta / \xi, & \kappa^{*}=R \kappa .
\end{array}
$$

We also define the ratio of the two characteristic length scales, the dimensionless average gap, as:

$$
\xi^{*}=\xi / R
$$

In addition, by introducing $\tau$, a characteristic time scale, the dimensionless time variable is written as $t^{*}=t / \tau$. Employing these length and time scales, the dimensionless thin-film equation describing the dynamics of the perturbed liquid film is given by:

$$
\begin{gathered}
\frac{\partial \eta^{*}}{\partial t^{*}}=\frac{1}{r^{*}} \frac{\partial}{\partial r^{*}}\left\{r^{*}\left[1+\frac{\xi^{*} \eta^{*}}{E^{*}}\right]^{3} \frac{\partial \Delta P^{*}}{\partial r^{*}}\right\}, \\
\Delta P^{*}=B_{\mathrm{o}} \eta^{*}+\frac{2}{\xi^{*}} \kappa^{*}+\frac{\hat{H} H_{\mathrm{a}}}{8 \xi^{*}\left(E^{*}\right)^{3}} \Pi_{\mathrm{ls}}^{*}+\frac{H_{\mathrm{a}}}{\xi^{*}} \Pi_{\mathrm{pl}}^{*} .
\end{gathered}
$$

where:

$$
\begin{gathered}
\kappa^{*}=-\frac{\xi^{*}}{2}\left\{\frac{1}{r^{*}} \frac{\partial}{\partial r^{*}}\left[r^{*} \frac{\partial \eta^{*}}{\partial r^{*}}\left\{\left[\xi^{*} \frac{\partial \eta^{*}}{\partial r^{*}}\right]^{2}+1\right\}^{-1 / 2}\right]\right\}, \\
\Pi_{\mathrm{pl}} *=\frac{-1}{\left\{\left[D^{*}-\xi^{*} \eta^{*}\right]^{2}+\left[r^{*}\right]^{2}-1\right\}^{3}},
\end{gathered}
$$

$$
\Pi_{\mathrm{ls}} *=-\left\{\left[1+\frac{\xi^{*} \eta^{*}}{E^{*}}\right]^{-3}-1\right\} .
$$

Moreover, three dimensionless parameters, which characterize the interface behavior, are yielded: the Bond number $B_{\mathrm{O}}=$ $\left[\rho g R^{2}\right] / \gamma$, the Hamaker constant ratio $\hat{H}=H_{\mathrm{ls}} / H_{\mathrm{pl}}$ and a modified Hamaker number $H_{\mathrm{a}}=4 H_{\mathrm{pl}} /\left[3 \pi \gamma R^{2}\right]$. Finally, for the consistency of the nondimensionalization process, the characteristic time scale results:

$$
\tau=\frac{3 \mu R^{4}}{\gamma E^{3}},
$$

which definition corresponds to the product of a classic capillary/ viscous time ${ }^{30-32} \tau_{\mathrm{c}}=\mu R / \gamma$ and the reciprocal of the cube of the dimensionless film thickness $E^{*}$. The value of $\tau$ indicates the time that the liquid film takes to move against the action of viscosity, when its surface is to be deformed. Therefore, depending on the liquid physical properties and the ratio of the film thickness to the probe size, with the use of eqn (13), one can infer the reaction time of the film. For a given liquid with known physical properties and a well characterized probe, one can conclude that a thick film, with a small $\tau$, displays a fast response, whereas a thin film, with a large $\tau$, provides a slow feedback.

Finally, the dimensionless probe periodic motion is given by the expression:

$$
D^{*}=\overline{D^{*}}+A^{*} \cos (\phi+2 \pi p)
$$

with:

$$
\begin{aligned}
\overline{D^{*}} & =\bar{D} / R, & A^{*} & =A / R, \\
\omega^{*} & =\omega \tau, & \phi & =\omega^{*} t^{*}-2 \pi p,
\end{aligned}
$$

where the phase takes values within the range $\phi \in[0,2 \pi]$ and the number of cycles is $p \in \mathbb{Z}^{+}$.

\section{Resolution method}

With the aim of performing a theoretical analysis, complemented by a numerical solution, we seek to reduce eqn (11). Considering small interface deformations $\xi^{*} \eta^{*} / E^{*} \ll 1$ and slopes $\xi^{*}\left[\partial \eta^{*} / \partial r^{*}\right] \ll 1$, hypotheses which were verified a posteriori, one is able to simplify the mean curvature, the liquidsubstrate term and the cubic term in the lubrication equation. The following quasi-linear partial differential equation, which depicts the evolution of the viscous thin-film interacting with a spherical probe, is deduced:

$$
\frac{\partial \eta^{*}}{\partial t^{*}}=\frac{1}{r^{*}} \frac{\partial}{\partial r^{*}}\left[r^{*} \frac{\partial \Delta P^{*}}{\partial r^{*}}\right]
$$

$$
\Delta P^{*}=-\frac{1}{r^{*}} \frac{\partial}{\partial r^{*}}\left[r^{*} \frac{\partial \eta^{*}}{\partial r^{*}}\right]+\frac{\eta^{*}}{\left[\lambda_{\mathrm{CF}}{ }^{2}\right.}+\frac{H_{\mathrm{a}}}{\xi^{*}} \Pi_{\mathrm{pl}} *,
$$

where the modified capillary length $\lambda_{\mathrm{CF}}{ }^{*}$, defined as ${ }^{11}$

$$
\lambda_{\mathrm{CF}} *=\left\{B_{\mathrm{o}}+\frac{3 \hat{H} H_{\mathrm{a}}}{8\left[E^{*}\right]^{4}}\right\}^{-1 / 2},
$$


appears. For a localized surface disturbance, which is radially transmitted due to surface tension effects, $\lambda_{\mathrm{CF}}{ }^{*}$ is the length scale at which the surface displacement is restrained by hydrostatic and substrate interaction effects. The nature and consequences of $\lambda_{\mathrm{CF}}{ }^{*}$ have been extensively discussed elsewhere. ${ }^{11,33}$

\subsection{Hankel transform}

Despite the previous assumptions, eqn (16) retains a nonlinear term, i.e. the probe-liquid interaction potential $\Pi_{\mathrm{pl}} *$. As it is crucial to understand the natural response of the thin-film to any perturbation, a theoretical analysis is devised. For this reason, we recall the Hankel transform of order zero (see Appendix A), which takes a variable defined in the spatial $r^{*}$ and temporal $t^{*}$ domains, and redefines it in the angular wavenumber $k^{*}=R k$ and time $t^{*}=t / \tau$ domains. The application of this transform turns the quasi-linear thin-film equation, given by eqn (16), into:

$$
\frac{\partial \mathscr{N}^{*}}{\partial t^{*}}=-\nu^{*} \mathscr{N}^{*}-\frac{H_{\mathrm{a}}}{\xi^{*}}\left[k^{*}\right]^{2} \mathscr{Q}^{*},
$$

where $\mathscr{N}^{*}$ and $\mathscr{Q}^{*}$ are:

$$
\begin{aligned}
& \mathscr{N}^{*}\left(k^{*}, t^{*}\right)=\mathbb{H}_{0}\left\{\eta^{*}\left(r^{*}, t^{*}\right)\right\}, \\
& \mathscr{Q}^{*}\left(k^{*}, t^{*}\right)=\mathbb{H}_{0}\left\{\Pi_{\mathrm{pl}} *\left(r^{*}, t^{*}\right)\right\},
\end{aligned}
$$

the Hankel transforms of the surface position and the probeliquid interaction, respectively. In addition, $\nu^{*}$ is defined as:

$$
\nu^{*}=\left[k^{*}\right]^{2}\left\{\left[k^{*}\right]^{2}+\left[\lambda_{\mathrm{CF}} *\right]^{-2}\right\},
$$

which is also identified as a wavenumber dependent time decay coefficient. This coefficient indicates that the relaxation time of a particular wavenumber $k^{*}$ is proportional to $\left[k^{*}\right]^{-4}$ for large wavenumbers (short wavelengths), whereas it scales as $\left[\lambda_{\mathrm{CF}} * / k^{*}\right]^{2}$ for relatively small wavenumbers (long wavelengths). In eqn (20), it is clear that $\left[\lambda_{\mathrm{CF}}{ }^{*}\right]^{-1}$ takes the role of a threshold wavenumber between the two behaviours.

\subsection{Numerical method}

The combination of eqn (12b) and (16) was solved with a homemade Fortran code, which employs a pseudo-spectral method. The implemented algorithm is based on the discrete Hankel and inverse Hankel transforms of order zero, which are computed in terms of Fourier-Bessel series, following a wellknown procedure. ${ }^{34}$ For instance, the Fourier-Bessel series of $\eta^{*}$ in terms of Bessel functions of the first kind and order zero is defined as:

$$
\eta^{*}\left(r^{*}, t^{*}\right)=\sum_{m=1}^{\infty} C_{m} J_{0}\left(\beta_{m} \frac{r^{*}}{\alpha_{\mathrm{r}} \lambda_{\mathrm{CF}}}\right)
$$

where $\beta_{m}$ is the $m^{\text {th }}$ root of $J_{0}(x)=0$. Using the definition of the Hankel transform (see eqn (34)), the Fourier-Bessel coefficients $C_{m}$ can be approximated by:

$$
C_{m} \simeq \frac{\mathscr{N}^{*}\left(\frac{\beta_{m}}{\alpha_{\mathrm{r}} \lambda_{\mathrm{CF}} *}, t^{*}\right)}{\pi\left[\alpha_{\mathrm{r}} \lambda_{\mathrm{CF}} J_{1}\left(\beta_{m}\right)\right]^{2}} .
$$

The radial position $r^{*}=\alpha_{\mathrm{r}} \lambda_{\mathrm{CF}}{ }^{*}$, with $\alpha_{\mathrm{r}} \in \mathbb{R}^{+}$, is the extent beyond which the film surface remains unperturbed.

Similarly, the Fourier-Bessel series of $\mathfrak{N}^{*}$ in terms of Bessel functions of the first kind and order zero is:

$$
\mathscr{N}^{*}\left(k^{*}, t^{*}\right)=\sum_{n=1}^{\infty} G_{n} J_{0}\left(\beta_{n} \frac{k^{*}}{\alpha_{\mathrm{k}} k_{\max }}\right),
$$

where, once more, $\beta_{n}$ is the $n^{\text {th }}$ root of $J_{0}(x)=0$. Using the definition of the inverse Hankel transform (see eqn (35)), the Fourier-Bessel coefficients $G_{n}$ are estimated from:

$$
G_{n} \simeq \frac{4 \pi \eta^{*}\left(\frac{\beta_{n}}{\alpha_{\mathrm{k}} k_{\max } *}, t^{*}\right)}{\left[\alpha_{\mathrm{k}} k_{\max }{ }^{*} J_{1}\left(\beta_{n}\right)\right]^{2}} .
$$

Herein, considering $\alpha_{\mathrm{k}} \in \mathbb{R}^{+}$, the wavenumber $\alpha_{\mathrm{k}} k_{\max }{ }^{*}$ designates a cutoff beyond which no other spatial frequencies are excited. The angular wavenumber $k_{\max } *$ has been obtained analytically from the coupling between the thin film equation and the probe-liquid interaction, and will be formally introduced in the following sections. Equivalent expressions were developed to calculate the discrete Hankel and inverse Hankel transforms of the probe-liquid interaction potential in space $\Pi_{\mathrm{pl}} *$ and wavenumber $\mathscr{Q}^{*}$ domains.

To briefly summarize, the implemented method starts with discrete Hankel transforms of the surface position and the probe-liquid interaction, which is obtained from the combination of discrete versions of eqn (14) and (12b); a temporal resolution in the wavenumber domain, using a first order semiimplicit Euler method to discretize eqn (18), follows in sequence; and finally, a discrete inverse Hankel transform is applied to obtain the surface evolution in the space domain.

\subsection{Parameters range}

Numerical solutions were obtained for $H_{\mathrm{a}}=5.5 \times 10^{-3}, B_{\mathrm{o}}=3.1$ $\times 10^{-11}, \hat{H}=1, E^{*}=1$ and $\tau=1.35 \times 10^{-7} \mathrm{~s}$. These parameters correspond to typical silicon oil (PDMS) physical properties $\gamma=$ $3.1 \times 10^{-2} \mathrm{~N} \mathrm{~m}^{-1}, \mu=1.4 \times 10^{-1} \mathrm{~Pa}$ s and $\rho=9.7 \times 10^{2} \mathrm{~kg} \mathrm{~m}^{-3}$, with a film thickness of $E=10^{-8} \mathrm{~m}$, generic silicon probes with $R=10^{-8} \mathrm{~m}$, and silicon probe/PDMS/silicon substrate interaction parameters $H_{\mathrm{pl}}=4 \times 10^{-20} \mathrm{~N} \mathrm{~m}$ and $H_{\mathrm{ls}}=4 \times 10^{-20} \mathrm{~N} \mathrm{~m}$, which are usually found in the literature or obtained from typical AFM experiments. ${ }^{9}$ Nevertheless, the present analysis can be extrapolated for other non-polar liquids (oils, liquid hydrocarbons and liquids consisting of diatomic molecules for instance), and ordinary AFM probes.

Considering the fixed aforementioned parameters, simulations were performed for different oscillation parameters. The angular frequency was varied within $\omega \in\left[2.2 \times 10^{5}, 2.2 \times 10^{7}\right]$ $\mathrm{s}^{-1}$. The time-average probe position was swept in the range $\bar{D} \in$ $(R+A, \infty)$, in order to analyse the impact of this parameter for a fixed $A$. The value $\bar{D}=R+A$ indicates contact between the probe, at its lower oscillation position, and the liquid surface, even without the deformation of the liquid surface. In turn, the oscillation amplitude was varied within $A \in(0,2 R]$. Therefore, 
the dimensionless ranges become $\omega^{*} \in\left[3 \times 10^{-2}, 3 \times 10^{0}\right]$, $\overline{D^{*}} \in\left(1+A^{*}, \infty\right]$ and $A^{*} \in(0,2]$.

In addition, we recall the dimensionless static minimum separation distance $D_{\min }{ }^{*}=D^{*} / R$, already introduced in the literature, ${ }^{11}$ which indicates the wetting threshold distance for the interaction with a static probe. For the employed dimensionless parameters, this threshold takes the value $D_{\min }{ }^{*}=$ 1.2017, which has been previously obtained ${ }^{11}$ with a precision of order $O\left(10^{-4}\right)$. Notice that the average position $\overline{D^{*}}=D_{\min }{ }^{*}+A^{*}$ is contained within the proposed range, which entails a probe lower position $\overline{D^{*}}-A^{*}=D_{\min }{ }^{*}$.

Since the problem has been solved using the aforementioned Hankel transform, discrete space (radial) and angular wavenumber domains were defined. A radial extent $\alpha_{\mathrm{r}} \lambda_{\mathrm{CF}}{ }^{*}$, with $\alpha_{\mathrm{r}}=$ 17.4 and $\lambda_{\mathrm{CF}}{ }^{*}=22.1$, and a cutoff wavenumber $\alpha_{\mathrm{k}} k_{\max }{ }^{*}$, with $\alpha_{\mathrm{k}}$ $=5.7$ and $k_{\max }{ }^{*}=4.4$, were selected. The values of $\alpha_{\mathrm{r}}$ and $\alpha_{\mathrm{k}}$ were chosen by a heuristic approach, verifying the convergence of the solution. The discretization procedure is based on the roots of a Bessel function of order zero, which are regularly spaced-out of $\beta_{n+1}-\beta_{n} \approx \pi$ for $n \gg 1$. Therefore, the discrete radial positions and angular wavenumbers are defined as $r_{n}{ }^{*}=$ $\beta_{n} /\left[\alpha_{\mathrm{k}} k_{\mathrm{max}}{ }^{*}\right]$ and $k_{\mathrm{m}}{ }^{*}=\beta_{m} /\left[\alpha_{\mathrm{r}} \lambda_{\mathrm{CF}}{ }^{*}\right]$, respectively. In addition, the number of meshpoints in both domains was fixed to $N=3071$.

Numerical convergence of the solution for the liquid surface position was tested, implying a relative error of order $O\left(10^{-3}\right)$. For $\overline{D^{*}}-A^{*} \geq D_{\min }{ }^{*}$, a time-step $\Delta t^{*}=10^{-2}$ was employed, whereas, for $\overline{D^{*}}-A^{*}<D_{\min }{ }^{*}$, a time-step $\Delta t^{*}=2 \times 10^{-3}$ was selected. Besides, we had arbitrarily chosen $p=200$ as the maximum number of cycles for the simulations.

\section{Results}

\subsection{Film surface dynamics}

In Fig. 2, a typical surface shape is shown at different instants of different oscillation cycles, using a phase-locking methodology that provides a long view of the surface evolution. During the first quarter of the oscillation period, from $\phi=0$ to (1), while the probe is relatively far from the surface, the liquid is quiescent, showing the shape acquired at the end of the previous cycle. In the case of the first oscillation cycle $p=0$, the shape is a flat profile. When the probe moves below its average position, up to (2), the probe-liquid interaction increases, provoking the formation of a small bump atop the former surface shape. When the probe reaches its lower position at (3), the bump is fed on the liquid that is drained from the immediate surroundings. Therefore, the bump surpasses the magnitude of the original surface profile, but presents a sharp contour. Afterwards, from (4) to (5), the probe retreat diminishes the probe-liquid interaction, inducing a decrease in the magnitude of the surface bump, together with a profile widening due to the liquid spreading. Note that, when comparing approach and retreat instants with the same probe distance (for instance, (2)/(4) and (1)/(5), the surface shape does not follow the same deformation path, and the downward motion of the surface during the probe retreat is slower than its upward displacement during the probe approach. Finally, at the end of the cycle (6), the liquid bump exhibits a wide shape with a slightly increased size, with respect to the one observed at the beginning of the oscillation period. A progressive accumulation of an important liquid amount below the probe position takes place, as it can be observed from the comparison between surface profiles of the same phase $\phi$ at different oscillation cycles $p$. Throughout the first 200 oscillations, in the transient regime for the case presented in Fig. $2 b$, the bump rises vertically from 0 to almost $10^{-2}$ times $R$, at the instant of maximum probe-liquid interaction, which is close to (3), and expands laterally up to the modified capillary length $\lambda_{\mathrm{CF}}$.

In Fig. 2c, the surface shape evolution shown in Fig. $2 b$ is portrayed in the wavenumber domain, using the same phaselocking methodology. When the probe is far from the liquid surface, from $\phi=0$ to (1), a bell-shaped distribution is observed in a range of relatively small wavenumbers $k^{*}<1$, which represents the remnant surface bump, due to the amassed liquid of the previous oscillation cycle. As the probe approaches, from (2) to (3), a second bell-shaped distribution appears and grows in magnitude, in a range of relatively large wavenumbers $k^{*}>0.2$. This secondary protuberance corresponds to the wavenumbers excited by the probe. At (3), the probe excited wavenumber distribution reaches its zenith shape, which is a direct consequence of the shortest probe-liquid separation distance. Afterwards, from (4) to (5), as the probe retreats and the probe-liquid interaction lessens, the secondary bell decreases in size and moves towards smaller wavenumbers, until it merges with the former small wavenumber distribution. Finally at (6), the wavenumber distribution regains its original single bell-shape, with a higher magnitude than the one observed at the beginning of the cycle. Throughout the entire phenomenon, the distribution grows slowly in magnitude and shifts towards smaller wavenumbers, as the number of oscillation cycles increases. This wavenumber mutation indicates the diffusion of deformation energy along gradually larger film regions, from $r^{*}$ $=1$ to $\lambda_{\mathrm{CF}}{ }^{*}$, which is coupled to the moderated film relaxation (drainage). In addition, the part of the distribution, which corresponds to the wavenumbers excited by the probe, attains quickly a stationary shape, overlaying almost exactly with one another at every oscillation cycle and indicating that the probeliquid interaction reaches a nearly periodic steady-state.

The surface evolution at $r^{*}=0$, defined as the surface apex $\eta_{0}{ }^{*}$, for the same conditions of the results displayed in Fig. 2, is shown in Fig. 3. This consists of a case for which the lower probe position $\overline{D^{*}}-A^{*}$ is larger than the threshold distance $D_{\min }{ }^{*}$, corresponding to the jump condition in a static probe situation. The transitory regime of $\eta_{0} *$ is displayed in Fig. 3a, for the first five oscillations with $p=0,1,2,3,4$, and in Fig. 3b, for the last cycle, with $p=199$. As the probe approaches the film, an abrupt increase of $\eta_{0}{ }^{*}$ occurs after an important deferring. Afterwards, when the probe moves away, a slow shrinkage of $\eta_{0}{ }^{*}$ is observed as a consequence of an unhurried film drainage. The increase of the $\eta_{0}{ }^{*}$ lower level, at the end of each oscillation, is also a consequence of this fact. In addition, even though the transitory regime is not entirely shown, the surface oscillation amplitude, defined as $W^{*}=\max \left(\eta_{0}{ }^{*}\right)-\min \left(\eta_{0}{ }^{*}\right)$, quickly attains a constant value, which occurs because the probe-liquid interaction reaches rapidly a steady-state. 

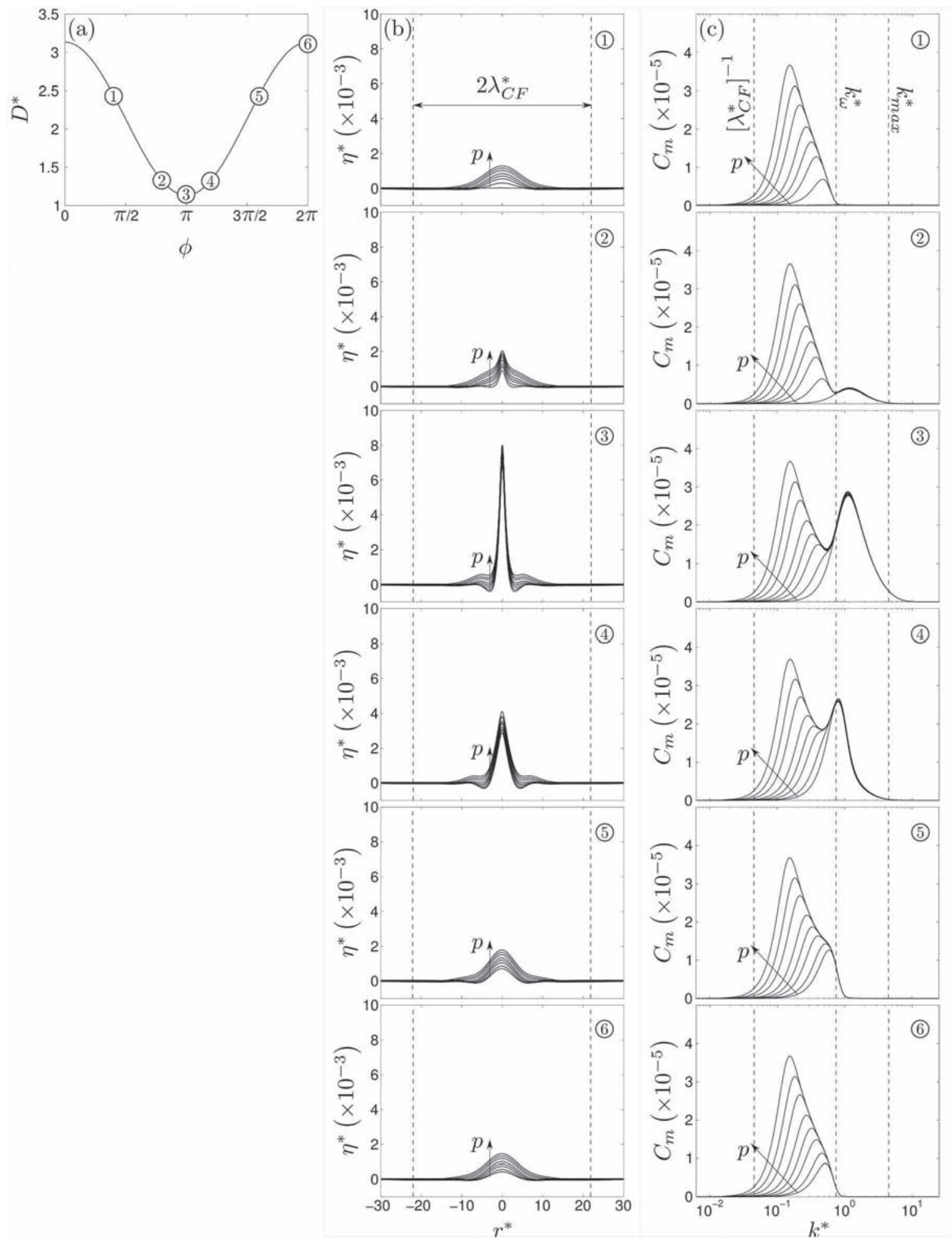

Fig. 2 (a) Probe center position $D^{*}$ as a function of the phase $\phi$, (b) surface vertical position $\eta^{*}$ as a function of the radial position $r^{*}$ and (c) Fourier-Bessel coefficients $C_{m}$ as a function of the wavenumber $k^{*}$. The figures in (b) and (c) correspond to the different instants with phase $\phi$, indicated in (a), and $p=0,1,4,9,19,49,99,199$ oscillation cycles, growing in the sense of the arrows. This surface evolution has been obtained for a probe time-average position $\overline{D^{*}}=2.2121$, a probe oscillation amplitude $A^{*}=1$ and an angular frequency $\omega^{*}=3 \times 10^{-1}$.

The terms given in eqn (16b), evaluated at $r^{*}=0$, are depicted and correlated with the evolution of $\eta_{0}{ }^{*}$ for the last oscillation cycle. The first quarter of the cycle is depicted by a film relaxation stage, in which the curvature is opposed only by the drainage effects. The remainder surface deformation from the previous cycle lessens due to the curvature restoring action, 


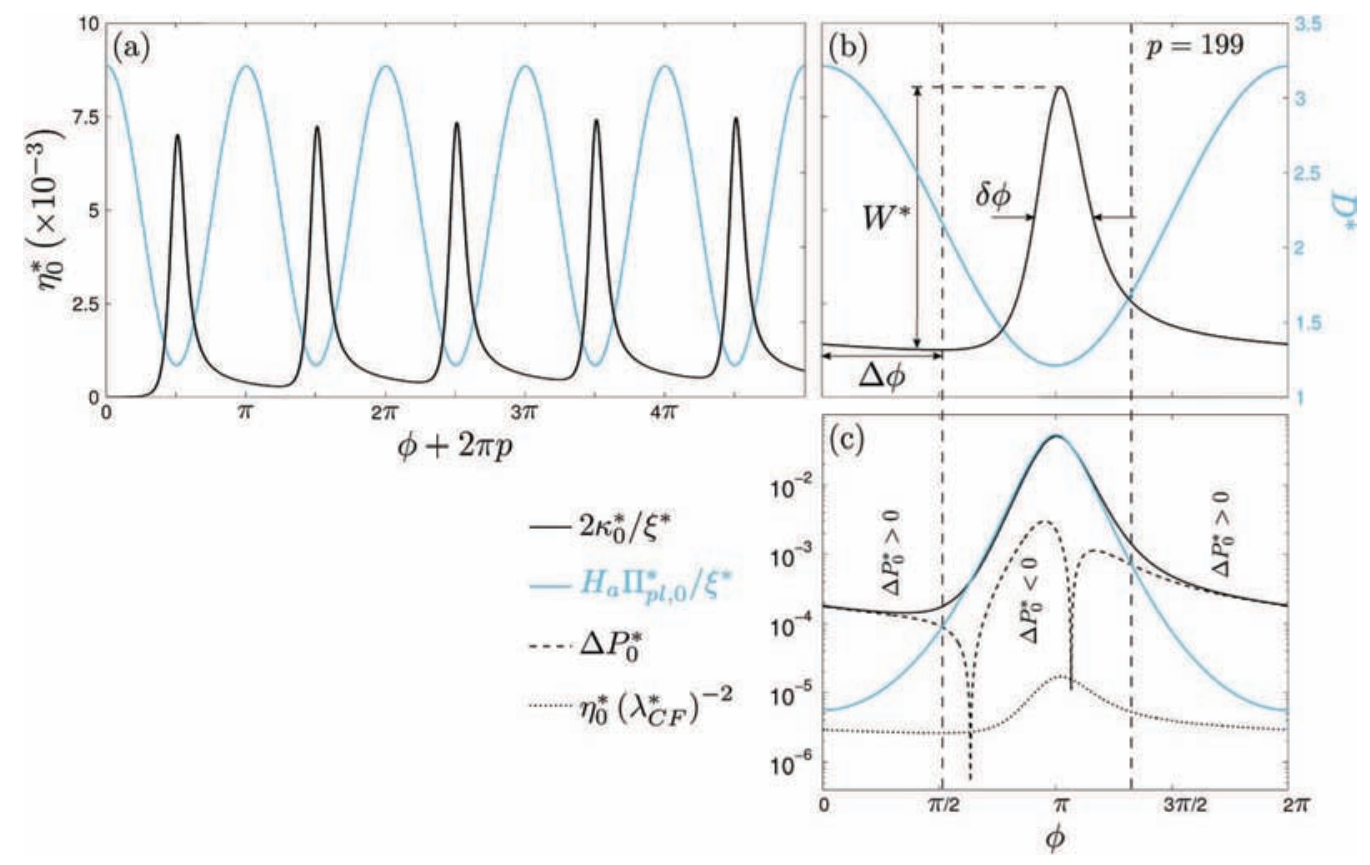

Fig. 3 Probe and apex surface positions for (a) the first 5 oscillation cycles and (b) the last computed period $p=199$, and (c) absolute value of the capillary, probe-liquid interaction, viscous drainage and hydrostatic terms, given in eqn (16b) for the aforementioned last period. The viscous drainage term changes its sign during each oscillation cycle, whereas the other terms keep their sense, because $\kappa_{0} *>0, \Pi_{\mathrm{pl}, 0} *<0$ and $\eta_{0} *>0$. The probe oscillation parameters correspond to the ones presented in Fig. 2.

the term containing $k_{0}{ }^{*}$ in Fig. $3 \mathrm{c}$. But the downward motion (relaxation) of the surface apex $\eta_{0} *$ is significantly opposed by the viscous drainage term $\Delta P_{0} *$. Additionally, the probe starts its motion far from the liquid surface, amplifying gradually its influence over the apex evolution. The hydrostatic-substrate interaction term $\eta_{0} *\left[\lambda_{\mathrm{CF}}{ }^{*}\right]^{-2}$ remains negligible in comparison with the other terms along the entire period. During the second quarter, the increase of $\eta_{0} *$ occurs abruptly, which marks the beginning of a strong probe-liquid interaction stage. In fact, this interaction, which quickly attains a dominant role, pulls up the surface, whereas the curvature term acts against the surface deformation. The drainage effects, which had become 10 times shorter in magnitude than the curvature and interaction terms, also resist faintly the upward motion of $\eta_{0}{ }^{*}$. The probe moves away from the film surface during the third quarter of the cycle, provoking the shrinkage of the $\Pi_{\mathrm{pl}, 0} *$ interaction term. The opposing curvature, which also decreases, becomes the dominant term and acts as a restoring force that pulls the surface towards its original non-deformed shape. At this moment, the film drainage turn its action against the curvature term, opposing the downward motion of the surface apex. The drop of $\eta_{0}$ * occurs rapidly at the beginning, until the interaction term is overtaken by the viscous drainage term, indicating the end of the probe-liquid interaction stage. Therefore, during the final quarter of the oscillation period, the film evolution becomes drainage-dominated and the apex slows down its downward motion. As the probe gets away, the $\Pi_{\mathrm{pl}}$ * interaction term loses completely its strength, and the curvature restoring action is only held by the film drainage term $\Delta P_{0}{ }^{*}$.
One can discern from Fig. $3 \mathrm{~b}$ that the periodic motions of the probe and the surface apex are globally in antiphase. The maximum apex position $\max \left(\eta_{0}{ }^{*}\right)$ and the lowest probe position $D^{*}=\overline{D^{*}}-A^{*}$ are almost synchronized (at $\phi=\pi$ ), which is consistent with a stage dominated by the balance between probe-liquid interaction and curvature. In contrast, the minimum value $\min \left(\eta_{0}{ }^{*}\right)$ is not concurrent with the farthest position $D^{*}=\overline{D^{*}}+A^{*}($ at $\phi=0)$, showing a phase shift of nearly $\Delta \phi \approx \pi / 2$. This phase delay $\Delta \phi$ is a characteristic fingerprint of a drainage effect, which provokes a time delay on the film reaction to the approaching probe. $\Delta \phi$ is thus defined as the phase difference between the moment at which the probe starts its downward motion and that at which the liquid film begins its upward displacement. Moreover, the phase fraction during which the probe liquid interaction is significant and mainly opposed by the surface curvature is approximately $9 \Delta \phi / 4$. Therefore, a wide $\Delta \phi$ indicates shorter periods of probe-liquid interaction and larger drainage stages, and vice versa. It is important to note that the alternation between interaction and drainage dominated stages only occurs in the vicinity below the probe position $r^{*} \in[0,1]$. For radial positions in the range $r^{*} \in$ $\left(1, \lambda_{\mathrm{CF}}{ }^{*}\right)$, only the balance between drainage and curvature effects is observed along the oscillation cycle, whereas for more distant positions beyond $r^{*}=\lambda_{\mathrm{CF}}{ }^{*}$, the hydrostatic effects take the place of drainage to resist the action of curvature.

In addition, based on the shape of the $\eta_{0}{ }^{*}$ curve in Fig. $3 \mathrm{~b}$, we introduce the full-width half-maximum $\delta \phi$, which points out the sharpness of the surface oscillation response. $\delta \phi$ gives rise to the classic finesse $F$ definition: 


$$
F=\frac{2 \pi}{\delta \phi}
$$

which has been taken from the optical interference theory. This quantity provides information about the way the deformation energy is distributed along the film surface. A large $F$ indicates an important transmission of deformation energy over a large area of the liquid surface throughout each oscillation cycle, whereas a short $F$ is found when the energy remains concentrated below the probe position.

\subsection{Probe oscillation amplitude and frequency effects}

In Fig. 4, the effect of the oscillation amplitude $A^{*}$ is studied, for fixed angular frequency $\omega^{*}$. The probe time-average position $\overline{D^{*}}$ and the oscillation amplitude $A^{*}$ were chosen so that the probe lower position is the same for all the cases $\overline{D^{*}}-A^{*}=1.2121$. The qualitative description of the apex position as a function of the phase during a period, for any considered case, corresponds to the same evolution as the one described in Fig. 3. Despite the qualitative similarity, important quantitative differences are discerned when comparing the evolution curves for different oscillation amplitudes. As $A^{*}$ decreases, and so does $\overline{D^{*}}$ to keep $\overline{D^{*}}-A^{*}$ constant, the probe-liquid interaction strengthens and, as a consequence, $\eta_{0} *$ maintains a higher level along the entire oscillation cycle. For smaller values of $A^{*}$, the curvature/interaction stage arrives earlier in a cycle, marked by a shorter $\Delta \phi$, which indicates that the probe-liquid interaction is faintly deferred by the film drainage. In addition, a long duration and strong interaction stage, together with a late beginning of the
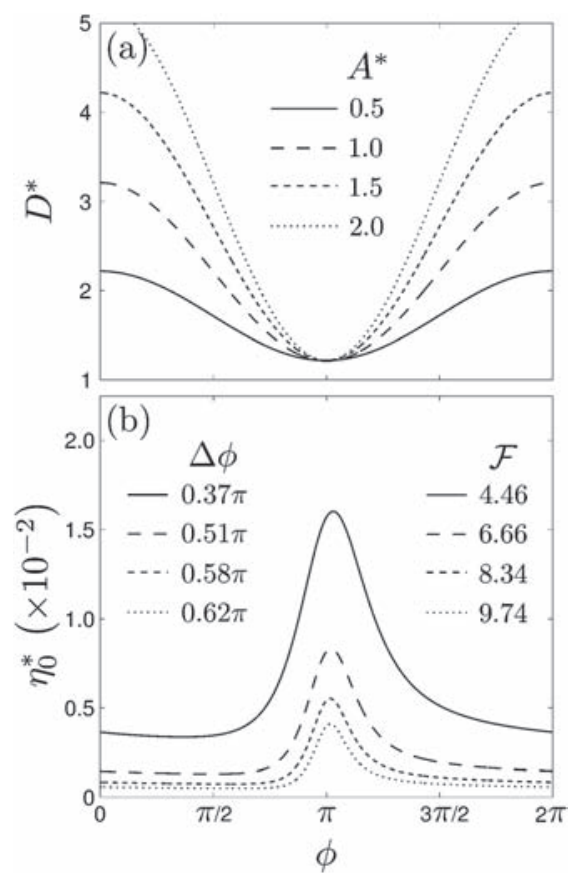

Fig. 4 (a) Probe position $D^{*}$, and (b) apex position $\eta_{0} *$, as a function of phase $\phi$. All the curves were obtained for the same angular frequency $\omega^{*}=3 \times 10^{-1}$ and probe lower position $\overline{D^{*}}-A^{*}=1.2121$, but for different values of the probe oscillation amplitude $A^{*}$. drainage stage, is observed for a small amplitude $A^{*}$, which is in accordance with a higher finesse $F$.

In Fig. 5, different angular frequencies $\omega^{*}$ are compared, whereas the time-average position $\overline{D^{*}}$ and the oscillation amplitude $A^{*}$ are fixed. As the probe trajectory is the same, whatever the frequency, so does the probe-liquid interaction term. Different values of $p$ are considered in Fig. 5, according to the angular frequency $\omega^{*}$, in order to compare oscillation cycles that start at the same time (except the quasi-static case), which in the presented case is $t^{*}=2 \pi p / \omega^{*}=421.8$. The surface apex evolution is symmetric with respect to $\phi=\pi$ for very low angular frequencies (typically $\omega^{*} \leq 10^{-6}$ ), corresponding to a quasi-static situation. For larger values of $\omega^{*}$, a symmetry breaking is induced by the gradually increasing role of the film relaxation (drainage). In addition, the maximum deformation $\max \left(\eta_{0}{ }^{*}\right)$ and the surface amplitude $W^{*}$ decrease, whereas the lower film position $\min \left(\eta_{0}{ }^{*}\right)$ grows, for higher angular frequencies $\omega^{*}$ (short oscillation periods). A more symmetric apex evolution provokes a shorter phase delay $\Delta \phi$ and a higher finesse $F$, which indicates that the curvature/interaction stage begins earlier and lasts longer during an oscillation cycle, and that the film relaxation occurs faster in relation to the probe motion (low oscillation frequency $\omega^{*}$ ). An asymmetric case denotes the opposite behaviour, as it can be clearly observed in Fig. 5.

\section{Wavenumber analysis}

\subsection{Cutoff wavenumbers}

In order to understand the behaviour of the liquid film, one seeks the range of wavenumbers which are involved in this
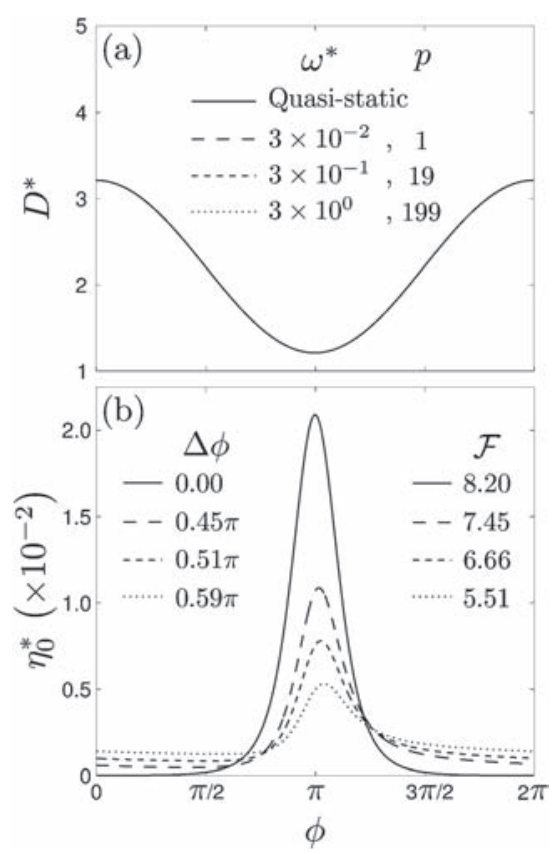

Fig. 5 (a) Probe position $D^{*}$, and (b) apex position $\eta_{0} *$, as a function of phase $\phi$. All the curves were obtained for the same time-average probe position $\overline{D^{*}}=2.2121$ and probe oscillation amplitude $A^{*}=1$, but for different values of the angular frequency $\omega^{*}$. 
phenomenon. The reciprocal of the modified capillary length $\left[\lambda_{\mathrm{CF}}{ }^{*}\right]^{-1}$ works as the lower cutoff wavenumber of an innate bandpass filter, since any distortion of the surface near $r^{*}=0$ tends naturally to propagate towards the modified capillary length. The upper cutoff, which is close to the wavenumber $k_{\max }{ }^{*}$, wherein the initial growth rate is maximum, is thus approximately:

$$
k_{\max } * \simeq 2 \pi\left[1+\frac{2-e_{0}}{e_{1} e_{D}}\right]
$$

where:

$$
\begin{aligned}
& e_{D}=2 \pi \sqrt{\left[D^{*}\right]^{2}-1}, \quad e_{0}=e_{D}\left[\frac{K_{1}\left(e_{D}\right)}{K_{2}\left(e_{D}\right)}\right] \\
& e_{1}=e_{D}\left\{\left[\frac{K_{1}\left(e_{D}\right)}{K_{2}\left(e_{D}\right)}\right]^{2}-\frac{K_{0}\left(e_{D}\right)}{K_{2}\left(e_{D}\right)}\right\}+2\left[\frac{K_{1}\left(e_{D}\right)}{K_{2}\left(e_{D}\right)}\right] .
\end{aligned}
$$

$K_{0}, K_{1}$ and $K_{2}$ are zero, first and second order modified Bessel function of the second kind. The maximization procedure of the wavenumber distribution at $t^{*}=0$, which leads to find $k_{\max }{ }^{*}$, is detailed in Appendix B. It is important to note that there is no relationship between $k_{\max }{ }^{*}$ and the other parameters appearing in eqn (18), i.e. the modified capillary length $\lambda_{\mathrm{CF}}{ }^{*}$ and the Hamaker number $H_{\mathrm{a}}$. The wavenumber $k_{\max }{ }^{*}$ that corresponds to the lower probe position $D^{*}=\overline{D^{*}}-A^{*}$ operates as the upper cutoff of the innate film band-pass filter. Furthermore, this particular value of $k_{\max }{ }^{*}$ has been employed as the cutoff wavenumber in the numerical method.

The cutoff wavenumbers $\left[\lambda_{\mathrm{CF}}{ }^{*}\right]^{-1}$ and $k_{\max }{ }^{*}$ have been presented in Fig. 2, proving the existence of a natural band-pass filter, which arises spontaneously from the physical and geometrical properties of the probe-film system. In other words, the structures that are observed at the film surface are always larger than $\left[k_{\max }{ }^{*}\right]^{-1}$ but shorter than $\lambda_{\mathrm{CF}}{ }^{*}$.

\subsection{Wavenumber dynamics}

Further understanding of the probe-film coupling can be achieved by assuming that $D^{*} \gg \xi^{*} \eta^{*}$, a small surface deformation compared to the separation distance, in eqn (12b). After applying the Hankel transform to the interaction potential, one finds $\mathscr{Q}^{*}=-\left[k^{*}\right]^{2} \mathscr{Q}_{\mathrm{s}}{ }^{*}$, with $\mathscr{Q}_{\mathrm{s}}{ }^{*}$ given by:

$$
\mathscr{Q}_{\mathrm{s}} *\left(k^{*}, D^{*}\right)=\frac{K_{2}\left(k^{*} \sqrt{\left[D^{*}\right]^{2}-1}\right)}{\left[D^{*}\right]^{2}-1} .
$$

The use of eqn (28) and a subsequent Fourier decomposition turns eqn (18) into a linear non-homogeneous ODE with analytical solution. This procedure, which is explained in-depth in Appendix C, yields the solution in the wavenumber domain:

$$
\begin{aligned}
\mathscr{N}^{*}= & \frac{\pi H_{\mathrm{a}}}{4 \xi^{*}}\left\{a_{0}\left[1-\exp \left(-\nu^{*} t^{*}\right)\right]\right. \\
& \left.+\sum_{j=1}^{\infty}\left[a_{j} \cos \left(j \omega^{*} t^{*}-\varphi_{j}\right)-\tilde{a}_{j} \exp \left(-\nu^{*} t^{*}\right)\right]\right\},
\end{aligned}
$$

where

$$
\begin{aligned}
& \tilde{a}_{j}\left(k^{*}\right)=\frac{a_{j} \nu^{*}}{\sigma_{j}}, \quad \sigma_{j}\left(k^{*}\right)=\sqrt{\left[j \omega^{*}\right]^{2}+\left[\nu^{*}\right]^{2}} \\
& \varphi_{j}\left(k^{*}\right)=\arctan \left(\frac{j \omega^{*}}{\nu^{*}}\right) .
\end{aligned}
$$

Eqn (29) provides an entire portrait of the wavenumber distribution dynamics and, as a consequence, the film surface evolution, although its Hankel transform must be obtained numerically. Additionally, a matching wavenumber $k_{\omega}{ }^{*}$ emerges from the comparison between $\nu^{*}$ and $\omega^{*}$. Using the definition given in eqn $(20), k_{\omega} *$ is given by:

$$
k_{\omega} *=\frac{1}{\sqrt{2}}\left\{-\left[\lambda_{\mathrm{CF}} *\right]^{-2}+\sqrt{\left[\lambda_{\mathrm{CF}} *\right]^{-4}+4 \omega^{*}}\right\}^{1 / 2} \text {. }
$$

The wavenumber-dependent coefficients $a_{0}$ and $a_{j}$, which are defined in Appendix $\mathrm{C}$ and can only be computed numerically, together with the coefficients $\tilde{a}_{j}$ and the phase $\varphi_{j}$, are shown in Fig. 6 as a function of the wavenumber $k^{*}$, wherein two different behaviours are discerned in respect of $k_{\omega}{ }^{*}$. For small wavenumbers $\left[\lambda_{\mathrm{CF}}{ }^{*}\right]^{-1}<k^{*}<k_{\omega}{ }^{*}$, the $a_{0}$ coefficient dominates over the others and a constant phase $\varphi_{j}=\pi / 2$ is found for any value of $j$. In other words, as it can be discerned from eqn (29), the surface shape contribution, given by wavenumbers $k^{*}<k_{\omega}{ }^{*}$, is not modified by the probe oscillation. Therefore, this wavenumber range portrays the transitory behaviour of the film surface, in the large time scale $t^{*} \sim\left[\lambda_{\mathrm{CF}}{ }^{*}\right]^{4}$. As $k^{*}$ takes values near the matching wavenumber $k_{\omega}{ }^{*}$, the first coefficients $a_{j}$ and $\tilde{a}_{j}$, obtained with small $j$ values, gain importance with respect to $a_{0}$. The phase $\varphi_{j}$ displays the same trend for any $j$, diminishing its value towards a zero phase as $k^{*}$ increases, and prematurely triggered for the large $j$ terms. For large wavenumbers, $k_{\omega}{ }^{*}<k^{*}<$ $k_{\max }{ }^{*}$, all the coefficients become as weighty as $a_{0}$, and a constant phase $\varphi_{j}=0$ is recovered for any $j$. Therefore, as deduced from eqn (29), the surface shape contribution displayed by wavenumbers $k^{*}>k_{\omega}{ }^{*}$ is completely driven by the probe oscillation. Thence, this range of large wavenumbers describes the periodic response of the film surface, in the short time scale $t^{*} \sim\left[\omega^{*}\right]^{-1}$.

Briefly, the film surface shape is partially described by a profile that saturates exponentially (given for $k^{*} \in\left[\left(\lambda_{\mathrm{CF}}\right)^{-1}\right.$, $\left.k_{\omega}{ }^{*}\right]$ ), due to the gradual amassing of liquid below the probe. This description is in agreement with the behaviour depicted in Fig. 2. In turn, along an oscillation cycle, the probe motion excites wavenumbers in the range $k^{*} \in\left[k_{\omega}^{*}, k_{\max }{ }^{*}\right]$, which provokes the surface oscillation around the saturation profile, spanning in the radial direction from the position beneath the probe to 15 times the probe radius. Since the time decay coefficient $\nu^{*}$ is a function of the wavenumber $k^{*}$, this probe excited wavenumber range, $k_{\omega}^{*}<k^{*}<k_{\max }{ }^{*}$, reaches a steady-state earlier than the left-hand side wavenumber distribution, $k^{*}<$ $k_{\omega}^{*}$.

It is important to note that for thin films (small $E^{*}$ and, consequently, shorter $\left.\lambda_{\mathrm{CF}}{ }^{*}\right)$ and higher frequencies $\omega^{*}$, the 


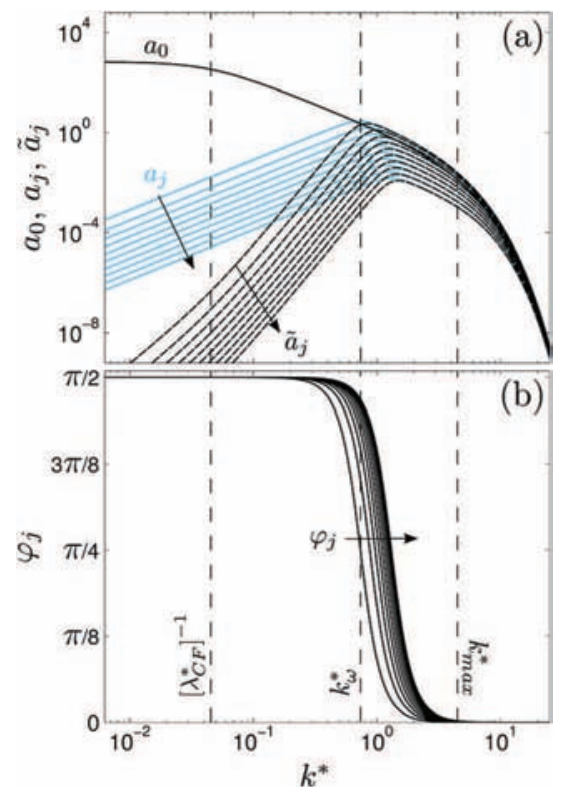

Fig. 6 (a) Coefficients and (b) phase of the solution given by eqn (29) for $\overline{D^{*}}=2.2121, A^{*}=1$ and $\omega^{*}=3 \times 10^{-1}$. The arrows indicate the terms corresponding to an increasing value of $j$.

matching wavenumber $k_{\omega}^{*}$ takes larger values. In this situation, the range of wavenumbers excited by the probe is narrowed, which also provokes a reduction in the radial extent of the film surface oscillation. The inverse effect should be produced for thick films (large $E^{*}$ and $\lambda_{\mathrm{CF}}{ }^{*}$ ) or lower frequencies $\omega^{*}$.

Since the evolution of $\mathscr{N}^{*}$ lies on the wavenumber-dependent coefficient $\nu^{*}$, a complete steady-state surface oscillation is reached only when time is comparable with the reciprocal of the lower cutoff wavenumber, i.e. $t^{*} \gg\left[\lambda_{\mathrm{CF}}{ }^{*}\right]^{4}$. Thenceforth, the stationary periodic regime is obtained when the exponential functions in eqn (29) are dismissed. For the case of a "slow" probe motion, which corresponds to a low frequency $\omega^{*} \ll$ $\left[\lambda_{\mathrm{CF}}{ }^{*}\right]^{-4}$, the matching wavenumber becomes $k_{\omega}^{*} \approx 0$, and the coefficients $\sigma_{j}$ and the phases $\varphi_{j}$ reduce to $\sigma_{j}=\nu^{*}$ and $\varphi_{j}=0$. This leads to the "slow" wavenumber distribution, which in the stationary state reduces to:

$$
\mathscr{N}^{*}=\frac{\pi H_{\mathrm{a}}\left[k^{*}\right]^{4}}{4 \xi^{*} \nu^{*}} \mathscr{Q}_{\mathrm{s}}^{*}\left(k^{*}, D^{*}\right)
$$

The liquid film has enough time to recover its nearly flat shape at the end of each oscillation cycle, which corresponds approximately to the static deformation obtained with the farther probe position $D^{*}=\overline{D^{*}}+A^{*}$. The film drainage occurs faster than the probe action, which inhibits the amassing of liquid and the increase of the film surface deformation. Therefore, the film oscillation process develops as a quasi-static phenomenon, revealing a surface shape that is equal to the static probe case for the same $D^{*}$, at any instant of the oscillation cycle. The proof of this fact is the behaviour of the apex deformation $\eta_{0}{ }^{*}$ during an oscillation, which is symmetric with respect to $\phi=\pi$, as shown in Fig. 7 .
In contrast, the probe motion is said to be "fast" when $\omega^{*} \gg$ $\left[k_{\max }{ }^{*}\right]^{4}$, which yields $\sigma_{j}=j \omega^{*}$ and $\varphi_{j}=\pi / 2$. Since $k_{\max }{ }^{*}>\lambda_{\mathrm{CF}}{ }^{*}$, therefore $k_{\omega}^{*} \sim\left[\omega^{*}\right]^{1 / 4}$, which implies that $a_{0}$ is dominant over any $a_{j}$. As a consequence, the "fast" wavenumber distribution for the stationary state is given by:

$$
\mathscr{N}^{*}=\frac{\pi H_{\mathrm{a}} a_{0}}{4 \xi^{*}} .
$$

In this situation, the liquid film does not have sufficient time to react to the probe oscillation. During the transitory regime, the film was not able to dissipate the energy injected at each cycle, which has been rather stored as an excess surface energy. Its amount is equivalent to the potential energy due to the liquid volume gathered near the probe position. Therefore, at the stationary state, the liquid surface remains with a "frozen-like" deformed shape, which is described by eqn (33). Since this expression only contains the constant term of the Fourier series of $\mathscr{Q}_{\mathrm{s}}{ }^{*}$, the surface shape does not correspond to a deformation profile generated by a static probe. Owing to the "fast" probe motion, the liquid film does not have enough time to spread the excess liquid volume, from the surroundings of $r^{*}=0$ towards the outer zone, to recover its flat profile, as it does for the quasistatic case. As it is shown in Fig. 7, the film surface keeps the same sharp shape (around $r^{*}=0$ ) along the entire oscillation cycle, as well as constant apex position $\eta_{0}{ }^{*}$ and a deformation extent that is shorter than $\lambda_{\mathrm{CF}}{ }^{*}$.

Also in Fig. 7, the surface apex position is shown for an oscillating probe with a frequency of $\omega^{*}=3 \times 10^{-1}$, corresponding to the intermediate frequency case shown in Fig. 5, which also occurs between the quasi-static and frozen-like behaviours. As it has already been mentioned, the apex evolution is not symmetric with respect to $\phi=\pi$ because of the

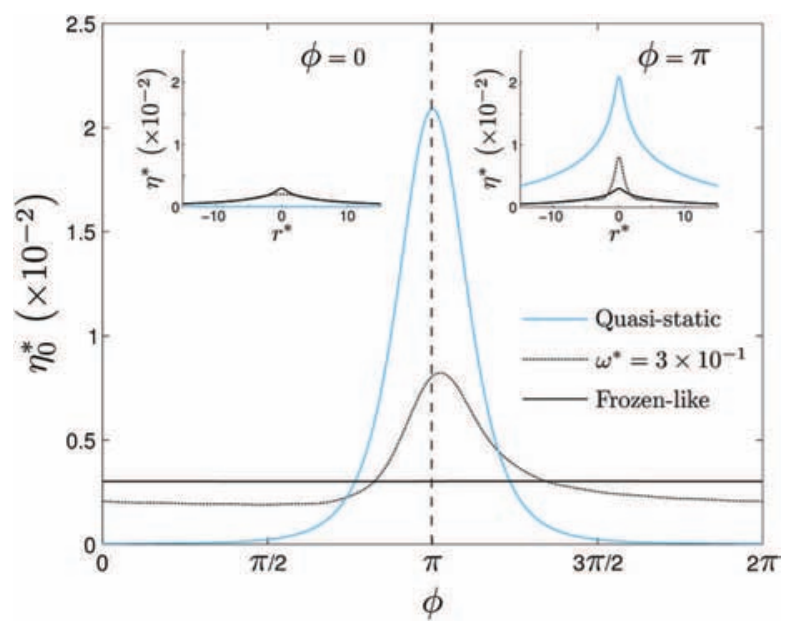

Fig. 7 Stationary state apex surface position $\eta_{0} *$ as a function of phase $\phi$, in the stationary regime. Insets display the surface position $\eta^{*}$ as a function of the radial position $r^{*}$ for $\phi=0$ and $\phi=\pi$. All the curves were obtained with $\overline{D^{*}}=2.2121$ and $A^{*}=1$. The quasi-static behaviour corresponds to eqn (32), in which $\omega^{*} \ll\left[\lambda_{\mathrm{CF}} *\right]^{-4}$, whereas the frozenlike behaviour is given by eqn (33), in which $\omega^{*} \gg\left[k_{\max }\right]^{4}$, and the intermediate steady-state case, $\omega^{*}=3 \times 10^{-1}$, is yielded by eqn (29) with $j=10$ coefficients. 
alternating dominant role between film drainage and probeliquid interaction. In addition, as it can be discerned from a comparison between eqn (29) and (33), a film surface, in the intermediate regime and the steady-state, oscillates around the frozen-like shape.

\section{Critical oscillation parameters and wetting transition}

Fig. 8 shows the surface vertical position and the Fourier-Bessel coefficients $C_{m}$ for different probe lower positions, above and below the value $\overline{D^{*}}-A^{*}=D_{\min }{ }^{*}$, at the instant of maximum probe-liquid interaction $\phi=\pi$. For relatively large distances $\overline{D^{*}}-A^{*} \geq D_{\min }{ }^{*}$, the film surface shows a narrow profile, below the probe position $r^{*} \in[0,2]$, surrounded by a not deep annular crater. Even though the vertical position of the liquid surface increases at each cycle, it remains far away from the probe lower surface. In addition, the direct effect of the probe-liquid interaction, represented by the large wavenumber side $k^{*}>k_{\omega}{ }^{*}$ of the $C_{m}$ distribution, quickly reaches a steady-state, opposite to the small wavenumber side $k^{*}<k_{\omega}^{*}$, which indicates the slow diffusion of deformation energy towards larger radial positions, before attaining the stationary regime. On the other hand, for shorter separation distances $\overline{D^{*}}-A^{*}<D_{\min }{ }^{*}$, the surface profile can never reach a steady-state, because the liquid jumpsto-contact the probe. The probe-liquid interaction increases at each oscillation, provoking the amassing of liquid below the probe position. Thus, the film surface exhibits progressively a more stretched profile, which becomes a vertical column of liquid at the last observable oscillation. At this last $p$ cycle, at which the $p$ value decreases along with the lower probe position $\overline{D^{*}}-A^{*}$, the liquid touches the probe surface. Under these circumstances, the $C_{m}$ distribution of the last period $p$ presents a large wavenumber side $k^{*}>k_{\omega}{ }^{*}$, that is considerably magnified with respect to the previous cycles, highlighting a significant augmentation of the probe-liquid interaction, which cannot attain a stationary regime. As observed when comparing Fig. $8 \mathrm{e}$ and $\mathrm{f}$, the probe wetting is characterized by a $C_{m}$ distribution of the same shape and magnitude in the wavenumber range $k^{*}>k_{\omega}^{*}$. As a consequence, the same deformation below the probe and a threshold intensity of the interaction are found, regardless of the number of cycles $p$ before the jump-to-contact occurs. Nevertheless, the value of $p$ indicates the periods that the system takes to attain this interaction frontier, due to the alternating attraction-relaxation stages. Therefore, a sequential transition from a stable surface oscillation regime with $p \rightarrow \infty$, observed for distant lower probe positions $\overline{D^{*}}-A^{*}>D_{\min }{ }^{*}$, to a delayed wetting phenomenon with $p>0$, for $\overline{D^{*}}-A^{*}<D_{\min }{ }^{*}$, and an instantaneous probe wetting event for $p=0$, for closer values $\overline{D^{*}}-A^{*} \ll D_{\min }{ }^{*}$, is discerned.

In Fig. 9, the surface oscillation amplitude $W^{*}$, the phase delay $\Delta \phi$ and the finesse $F$, already defined in Fig. 3 and eqn (25), are shown as a function of the number of oscillation cycles $p$ and for a probe oscillation amplitude $A^{*}=1$. For relatively large lower distances $\overline{D^{*}}-A^{*} \geq D_{\min }{ }^{*}$, a stationary state is pursued, and thus $W^{*}$ and $\Delta \phi$ reach a saturation value after the transient regime, consisting of several oscillation cycles $p \approx 25$, whereas $F$ slowly converges towards a constant level. The final stage of $W^{*}, \Delta \phi$ and $F$ becomes higher as $\overline{D^{*}}-A^{*}$ diminishes and approaches $D_{\min }{ }^{*}$. For slightly shorter distances $0.95 D_{\min }{ }^{*}<\overline{D^{*}}-A^{*}<D_{\min }{ }^{*}$, after the initial stage of the
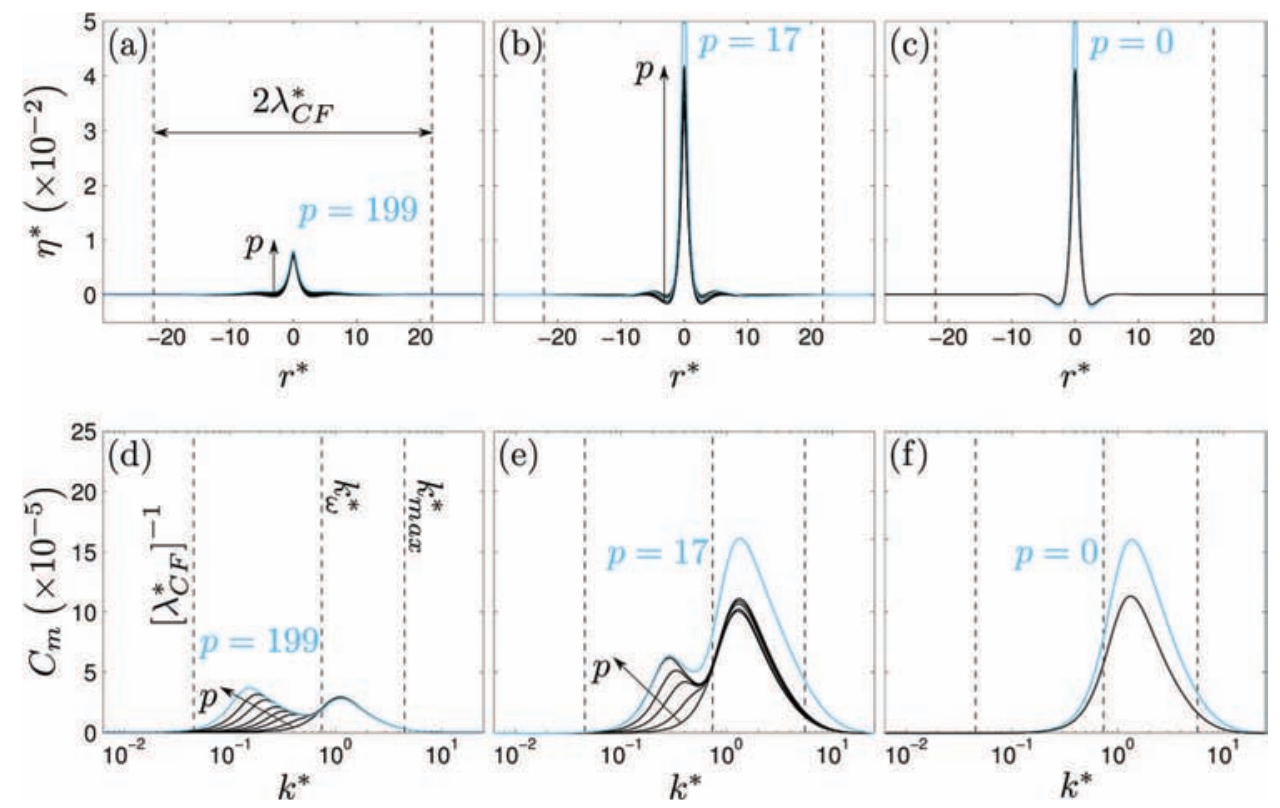

Fig. 8 (a-c) Surface vertical position $\eta^{*}$ as a function of the radial position $r^{*}$ and (d-f) Fourier-Bessel coefficients $C_{m}$ as a function of the wavenumber $k^{*}$, for (a and d) $\overline{D^{*}}-A^{*}=1.009 D_{\min }{ }^{*}$, a non-wetting regime, (b and e) $\overline{D^{*}}-A^{*}=0.948 D_{\min } *$, a delayed wetting, and (c and f) $\bar{D}^{*}-A^{*}=0.945 D_{\min }{ }^{*}$, an instantaneous wetting phenomenon. All the curves were obtained for $A^{*}=1$ and $\omega^{*}=3 \times 10^{-1}$, and they correspond to the phase $\phi=\pi$. 


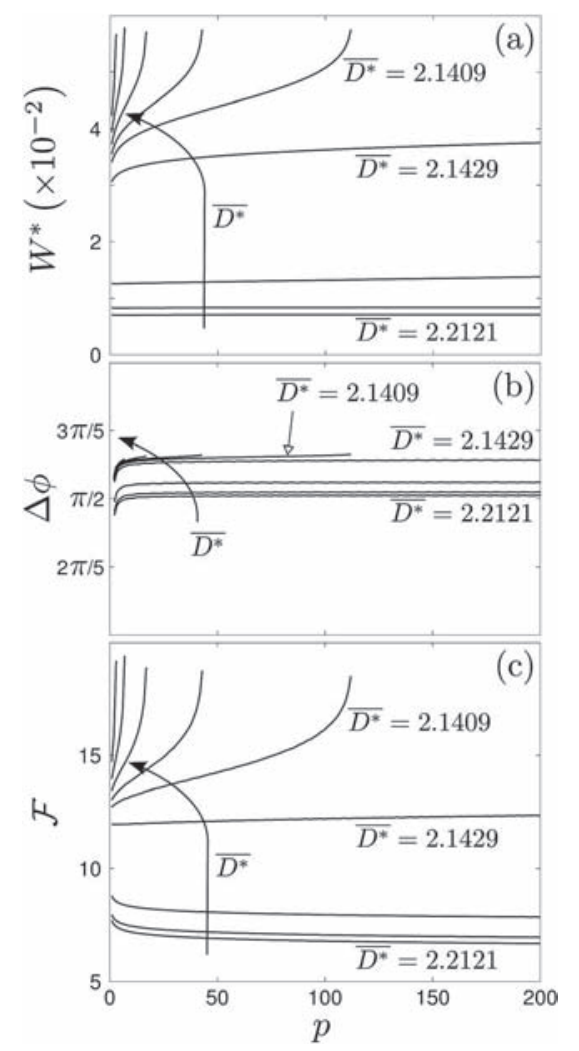

Fig. 9 (a) Surface oscillation amplitude $W^{*}$, (b) phase delay $\Delta \phi$ and (c) finesse $F$ as a function of the number of cycles $p$. All the curves were obtained for the same angular frequency $\omega^{*}=3 \times 10^{-1}$ and probe oscillation amplitude $A^{*}=1$, but for different time-average probe positions in the range $\overline{D^{*}} \in[2.1383,2.2121] . \overline{D^{*}}$ decreases in the sense of black arrows.

transient regime, the growing rates of $W^{*}, \Delta \phi$ and $F$ tend to stabilize, although, they slowly continue to increase with faint slopes. For $\overline{D^{*}}-A^{*}<0.95 D_{\min }{ }^{*}$, the surface amplitude $W^{*}$ and the finesse $F$ curves look like inverse hyperbolic tangents. These quantities always increase, suffering from an important decrease in the growth rate during the first cycles and reaching a minimum slope, and then, as the growth rate becomes unbounded again, $W^{*}$ and $F$ are amplified until they diverge near a vertical asymptote. In turn, for these relatively small probe lower distances $\overline{D^{*}}-A^{*}$, the phase delay $\Delta \phi$ increases strongly during the first oscillations, which corresponds to an enhancement of the film drainage effects and an important surface deformation along each oscillation cycle. Afterwards, as the cycles go by, $\Delta \phi$ calms down and its growth rate shows more gentle slopes, until it reaches a critical value $\Delta \phi \sim 0.58 \pi$, wherein it completely halts.

The divergence of $W^{*}$ and $F$, together with the halt of $\Delta \phi$, indicates the wetting of the probe by the liquid film depending on the lower probe position $\overline{D^{*}}-A^{*}$. In Fig. 10, typical phase spaces, of the apex position $\eta_{0}{ }^{*}$ and the separation distance $D^{*}$, are shown for different values of $\overline{D^{*}}-A^{*}$. For $\overline{D^{*}}-A^{*}=1.01 D_{\min }{ }^{*}$, the liquid film reaches the oscillatory steady-state, a non-wetting behaviour, and thus a limit cycle attractor is observed in the phase space. This limit periodic

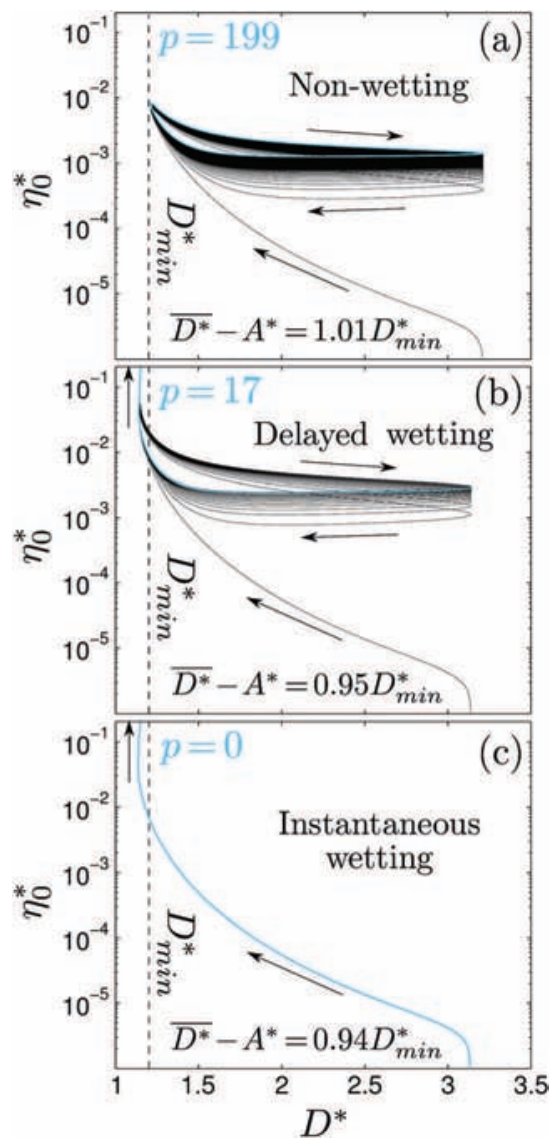

Fig. 10 Phase diagram of the apex position $\eta_{0} *$ and the separation distance $D^{*}$ of the three different behaviours: (a) non-wetting for $\overline{D^{*}}-A^{*}=1.01 D_{\min } *$, showing a transient regime and approaching a limit periodic orbit in the permanent state, (b) delayed wetting for $\overline{D^{*}}-A^{*}=0.95 D_{\min } *$, showing a similar transient regime but diverging from a possible limit orbit at the half-period of the $p=17$ cycle, and (c) instantaneous wetting for $\overline{D^{*}}-A^{*}=0.94 D_{\min } *$, showing a monotonic trajectory that diverges immediately at $p=0$. Curves obtained with $A^{*}=1$ and $\omega^{*}=3 \times 10^{-1}$.

trajectory, of $\eta_{0}{ }^{*}$ as a function of $D^{*}$, exhibits a "chistera" shape, following a clockwise motion with smooth slopes around $D^{*}=\overline{D^{*}}+A^{*}$ and a stepper path near $D^{*}=\overline{D^{*}}-A^{*}$. This particular pattern is shown in Fig. 10a, up to $p=199$ oscillation cycles. For $\overline{D^{*}}-A^{*}=0.95 D_{\min } *$, a delayed probe wetting of $p=$ 17 cycles is observed. In this case, although the apex displacement seems to reach a periodic orbit, its trajectory shifts constantly towards greater values of $\eta_{0}{ }^{*}$. As a consequence of this gradual augmentation, the probe-liquid interaction becomes unbounded and the liquid rises to touch the probe, at the half-period of the $p=17$ cycle in Fig. 10b. Finally for $\overline{D^{*}}-A^{*}=0.94 D_{\min }{ }^{*}$, the liquid film touches the probe at the first oscillation cycle, as it is shown in Fig. 10c. Therefore, at the half-period of the first cycle $p=0$, the instant of maximum probe-liquid interaction, an instantaneous wetting process occurs after a single barely curved trajectory of the liquid film apex $\eta_{0}{ }^{*}$. In brief, the liquid film wets the probe for $\overline{D^{*}}-A^{*}<D_{\min } *$ at a limit number of cycles $p$, which lessens as the probe lower position $\overline{D^{*}}-A^{*}$ is shortened. 


\section{AFM experimental consequences}

The results presented in this theoretical study correspond to a typical AFM-film system, consisting of a silicon probe oscillating near a liquid PDMS film placed over a silicon wafer (substrate), with specific physical and geometrical properties and a single combination of dimensionless parameters $\left(H_{\mathrm{a}}, B_{\mathrm{o}}\right.$, $\left.\hat{H}, E^{*}\right)$, which has been already given in Section 3.3. The effect of these dimensionless numbers can be observed and analysed through their relationship with the merging length, time and wavenumber scales (see eqn (13) and (17) and Fig. 2): modified capillary length $\lambda_{\mathrm{CF}}=2.2 \times 10^{-7} \mathrm{~m}$, film time scale $\tau=1.35 \times$ $10^{-7} \mathrm{~s}$, transitory regime duration $t \sim \tau\left[\lambda_{\mathrm{CF}} / R\right]^{4}=3.2 \times 10^{-2} \mathrm{~s}$ and lower cutoff wavenumber $k=\left[\lambda_{\mathrm{CF}}\right]^{-1}=4.5 \times 10^{6} \mathrm{~m}^{-1}$. In addition, we recall the static threshold $D_{\text {min }}$, the separation distance below which the liquid jumps-to-contact a static probe, which for the aforementioned parameters is $D_{\min }=$ $1.2017 \times 10^{-8} \mathrm{~m}$.

In contrast, different combinations of the probe oscillation parameters have been presented. The time-average probe position $\bar{D}$ and oscillation amplitude $A$ influence directly the film surface oscillation amplitude $W$, the probe wetting conditions and the corresponding limit of oscillation cycles $p$ (see Fig. 4, 9 and 10). In addition, the impact of the probe oscillation frequency $\omega$ lies on the wavenumber scales: upper cutoff wavenumber $k_{\max }$ and matching wavenumber $k_{\omega}$ (see eqn (38) and (31)). It is important to remember that these two quantities delimit the range of wavenumbers excited by the approach of the probe. Considering the aforementioned probe/film/ substrate system and common dynamic NC-AFM tests, with frequencies in the range of $10^{1}$ to $10^{3} \mathrm{kHz}$, oscillation amplitudes and probe-liquid separation distances restricted to the range of 1-25 nm, the probe activates a wavenumber range with lower $k_{\omega} \in\left[10^{7}, 10^{8}\right] \mathrm{m}^{-1}$ and upper $k_{\max } \in\left[10^{8}, 10^{9}\right] \mathrm{m}^{-1}$ boundaries.

This phenomenon can be analysed from an analogous viewpoint, performing a comparison between the two main time scales: the reciprocal of the angular frequency $\omega^{-1}$, corresponding to the experimental AFM time scale, and the characteristic film time scale $\tau$, which also refers to the film relaxation time. ${ }^{30}$ Therefore, the dimensionless angular frequency $\omega^{*}=\omega \tau$ is also identified as the Deborah number $D_{\mathrm{e}}$ $=\omega \gamma E^{3} / 3 \mu R,{ }^{4}$ which herein characterizes the liquid film response to a periodic AFM nano-probe periodic excitation. Large Deborah numbers $D_{\mathrm{e}}$ are obtained for liquids of high viscosity $\mu$ or relatively thin films $E$, which correspond to large relaxation times $\tau$. This situation is also discerned when the film is perturbed by a probe oscillating with a high frequency $\omega \gg\left[R k_{\max }\right]^{4} / \tau$, where $k_{\max }$ depends on the lower probe position $\bar{D}-A$. This high frequency case also yields a relatively large matching wavenumber, approximately $k_{\omega} \simeq R$ $[\omega \tau]^{1 / 4}$. In this large $D_{\mathrm{e}}$ regime, the film time scale $\tau$ governs the dynamics of the liquid surface. Since the surface $\eta(r, t)$ evolves during the transient regime, finally reaching a steady-state $\eta(r)$ independent of time $t$ (see Fig. 7), a frozen-like behaviour of the film is observed. Therefore, all the terms in eqn (11) become constant over time. The viscous drainage restrains and slows down the film dynamics, which coherently provokes a phase delay $\Delta \phi \rightarrow \pi$. As indicated by the observed finesse $F \rightarrow 2$, the surface deformation is restrained to a span shorter than the modified capillary length $\lambda_{\mathrm{CF}}$, and to relatively small vertical displacements. The deformation energy is gathered below the probe position, generating a very narrow surface profile, due to the slow film relaxation (drainage). Considering the probefilm-substrate system analysed in this work, this frozen-like state should be observed for a probe oscillating around an average position $\bar{D} \leq 35 \mathrm{~nm}$ with an amplitude of $A=10 \mathrm{~nm}$, only at frequencies above $10 \mathrm{MHz}$, which is only achieved with ultra-high frequency probes for high-speed AFM.

Small Deborah numbers $D_{\mathrm{e}}$ are found for liquids of low viscosity $\mu$ or relatively thick films $E$, which yields short relaxation times $\tau$. Equivalent similarity conditions are also generated by low probe oscillation frequencies $\omega \ll 1 / \tau\left[\lambda_{\mathrm{CF}} / R\right]^{4}$, which for our specific thin film corresponds to $\omega \ll 3.1 \times 10^{1} \mathrm{~s}^{-1}$, leading to a matching wavenumber $k_{\omega}=0$. In this small $D_{\mathrm{e}}$ regime, the AFM experimental time scale $[\omega]^{-1}$ determines the film evolution, displaying a quasi-static behaviour. The viscous drainage occurs quickly and a full-period probe-liquid interaction is observed, leading to a fast and full film reaction, which corresponds to a phase delay $\Delta \phi \rightarrow 0$. At each instant, the surface attains the equilibrium shape of a static probe-liquid interaction phenomenon, with a maximum vertical displacement and a deformation extent that covers $\lambda_{\mathrm{CF}}$. Therefore, the drainage term in eqn (11) becomes negligible, and the probeliquid interaction is only opposed by the curvature term throughout an oscillation cycle. This behaviour is validated by a large valued finesse $F$, pointing out that the deformation energy is spread over a large radial span, owing to a relatively rapid film relaxation (drainage). For the parameters of the proposed probe-film-substrate system, this regime is perceived for a probe oscillating with a frequency lower than $5 \mathrm{~Hz}$, which is hardly considered as a dynamic AFM mode. Note that this frequency increases as the thickness of the film is reduced. For instance, for a film of $1 \mathrm{~nm}$, the quasi-static threshold frequency is $5 \times 10^{5} \mathrm{~Hz}$, which means that almost the entire NC-AFM operation range presents a quasi-static behaviour. This last case must be considered for example when a thin layer of water is adsorbed over a silicon wafer. ${ }^{1,15}$

A comparison between the different $D_{\mathrm{e}}$ regimes, corresponding to a thin film of $10 \mathrm{~nm}$, and several AFM modes, showing their frequency $\omega$ range, is depicted in Fig. 11. The upper limit of the quasi-static behaviour, which is fixed for a given film thickness, also defines the limit of force spectroscopy tests, whereas the lower boundary of the frozen-like behaviour moves according to the probe lower position. In the presented diagram, normal NC-AFM experiments occur in the transition zone, above the quasi-static boundary, which indicates that the probe oscillation provokes a significant surface oscillation amplitude and a transmission of surface energy to a large radial extent, but not as important as for the quasi-static case. The frozen-like boundary is located within the higher frequency range of high-speed NC-AFM experiments, implying surface profiles with small surface oscillation amplitudes and 
AFM experiments

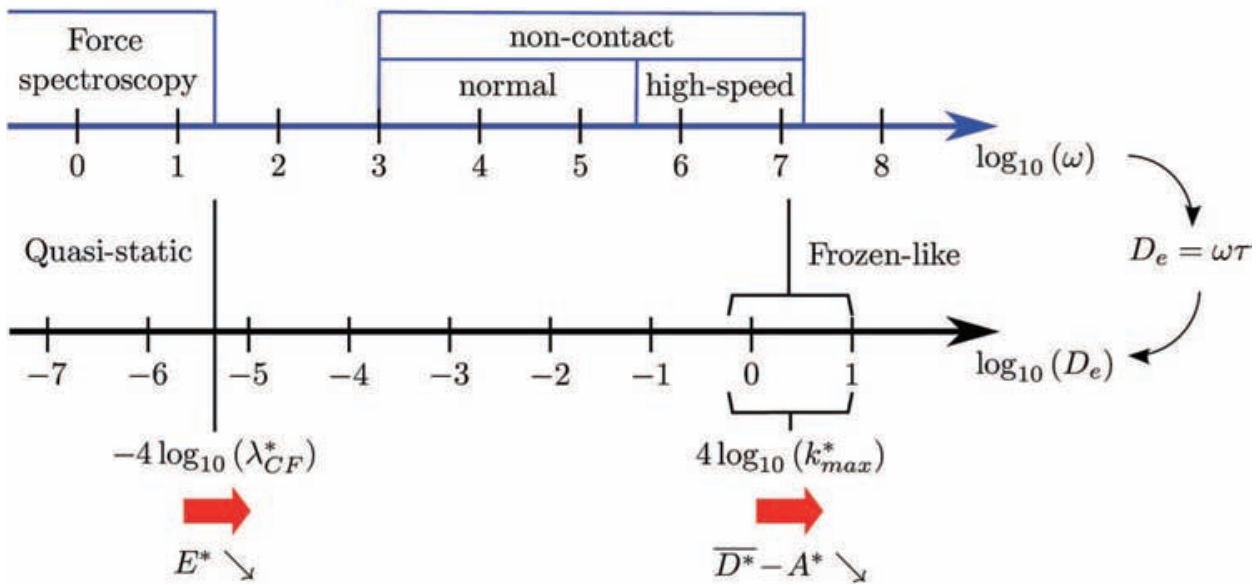

Fig. 11 AFM modes and liquid film behaviour depending on the Deborah number $D_{\mathrm{e}}$. The boundaries for the quasi-static and frozen-like behaviour were placed according to their values for a film thickness of $10 \mathrm{~nm}$ and a lower probe position $1<\overline{D^{*}}-A^{*}<3.5$. Arrows (red) indicate the impact of decreasing the parameters: film thickness $E^{*}$ and the lower probe position $\overline{D^{*}}-A^{*}$.

restrained radial spans, under these conditions. Although an equivalent diagram can be obtained for a film of different thickness, a particular interpretation of the AFM modes should be done due to the thickness-dependence of $D_{\mathrm{e}}, \tau$ and $\lambda_{\mathrm{CF}}$. For instance, considering a film with a thickness of $1 \mathrm{~nm}$, a large range of normal NC-AFM experiments may be comprised in the quasi-static regime. On the other hand, the quasi-static state is never observed for NC-AFM tests over a film of $100 \mathrm{~nm}$, because the frequency needed to reach this situation becomes five orders of magnitude smaller than that for the $10 \mathrm{~nm}$ film case.

In Fig. 12, for a fixed oscillation amplitude $A^{*}=1$, the limit number of oscillations before wetting $p$ is shown as a function of $\overline{D^{*}}-A^{*}-D_{\min } *$ the difference between the lower probe position and the static minimum separation distance, also corresponding to the threshold jump-to-contact distance of a quasi-static situation. The trend for an intermediate angular frequency $\omega^{*}=3 \times$ $10^{-1}$, which is located in the intermediate Deborah regime $\left[\lambda_{\mathrm{CF}}{ }^{*}\right]^{-4}<D_{\mathrm{e}}<\left[k_{\max }{ }^{*}\right]^{4}$, is shown in Fig. 12 . The instantaneous probe wetting occurs for $\overline{D^{*}}-A^{*}<D_{\min }{ }^{*}-0.066$, whereas a delayed wetting behaviour is observed within the zone $-0.066<\overline{D^{*}}-A^{*}-D_{\min } *<-0.057$, and the oscillating steadystate without wetting takes place for $\overline{D^{*}}-A^{*}<D_{\min }{ }^{*}-0.057$. These three situations, which correspond to previously mentioned behaviours (see Fig. 10), are connected by a monotonically increasing dependency of $p$ on $\overline{D^{*}}-A^{*}-D_{\min }{ }^{*}$. Interestingly, the non-wetting transition does not take place at $\overline{D^{*}}-A^{*}=D_{\min }{ }^{*}$, the jump-to-contact threshold distance for a static probe. In addition, the wetting transition for the two asymptotic frequency cases is also depicted in Fig. 12, for $A^{*}=1$. The right-hand side inlet in Fig. 12 corresponds to a small Deborah regime $D_{\mathrm{e}} \ll\left[\lambda_{\mathrm{CF}}{ }^{*}\right]^{-4}$ (quasi-static film behaviour), in which wetting may occur instantaneously for $\overline{D^{*}}-A^{*}<D_{\min }{ }^{*}$, following the same trend as the phase diagram in Fig. 10c with $p=0$. Under these conditions, an oscillating steady-state should always be observed for $\overline{D^{*}}-A^{*} \geq D_{\min }{ }^{*}$, equivalent to the periodic trajectory shown in Fig. 10a. A delayed wetting event can never occur for the quasi-static situation, and a straightforward transition from wetting to non-wetting is observed, displayed as a vertical asymptote overlapping the abscissa $\overline{D^{*}}-A^{*}-D_{\min }{ }^{*}=0$ in the right side inlet in Fig. 12. Dynamic NC-AFM experiments executed in this small $D_{\mathrm{e}}$ regime are restricted to large separation distances $\bar{D}-A>D_{\mathrm{min}}$, which reduces significantly the probe sensitivity, in order to inhibit the probe wetting. On the other hand, the large Deborah regime $D_{\mathrm{e}} \gg\left[k_{\max }{ }^{*}\right]^{4}$ (frozen-like film behaviour) is displayed in the left-hand side inlet of Fig. 12. In this case, wetting is reprieved to shorter separation distances and a vertical asymptote placed at $\overline{D^{*}}-A^{*}-D_{\min }{ }^{*}=-0.153$ indicates the wetting transition. This threshold distance has been

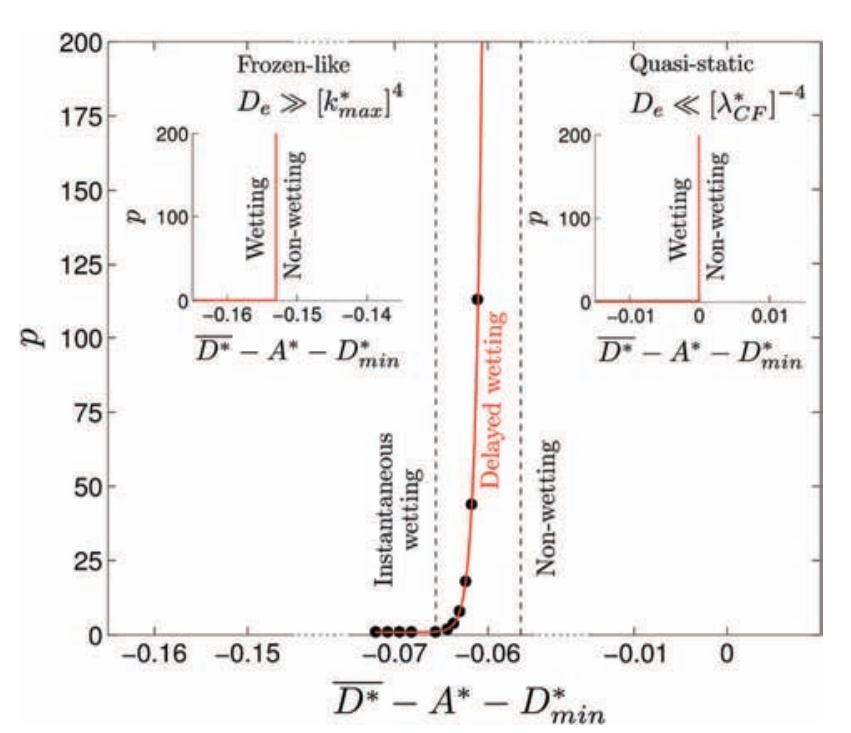

Fig. 12 Number of oscillation cycles $p$ before wetting as a function of the difference between the lower probe position $\overline{D^{*}}-A^{*}$ and the static minimum separation distance $D_{\min } *$, obtained with $A^{*}=1$ and $\omega^{*}=3$ $\times 10^{-1}$, within an intermediate Deborah regime $\left[\lambda_{\mathrm{CF}}{ }^{*}\right]^{-4}<D_{\mathrm{e}}<\left[k_{\max } *\right]^{4}$. The quasi-static and frozen-like trends, including their wetting thresholds, are also depicted. 
obtained by solving $\overline{D^{*}}-A^{*}=\xi^{*} \eta_{0}{ }^{*}+1$, with the use of eqn (33). Therefore, when dynamic NC-AFM experiments are performed in this large $D_{\mathrm{e}}$ regime, the liquid film surface can be scanned at shorter probe-liquid separation distances $\bar{D}-A<$ $D_{\text {min }}$, which increases the apparatus resolution without the risk of wetting the probe or engendering a significant surface deformation.

When performing NC-AFM experiments over liquids, one must prevent the sample damage and the probe wetting. The employment of a high oscillation frequency (large $D_{\mathrm{e}}$ regime) allows "freezing" of the dynamic response of the liquid film, which generates the following advantages:

(1) The probe-liquid separation distance can be shortened, increasing the AFM sensitivity and preserving the sample physical integrity. In the presented case, this distance, which corresponds to the probe lower position $\overline{D^{*}}-A^{*}$, can be shortened by around $1.5 \mathrm{~nm}$ (see Fig. 12), around 13\% of the static threshold separation distance ${ }^{11} D_{\text {min }}$.

(2) The amplitude of the liquid surface oscillation is small, thus the effect of its displacement over the measured topography is reduced. The maximum deformation of the surface is reduced up to $86 \%$, from the quasi-static to the frozen-like regime (see Fig. 7).

(3) The surface oscillation is restrained and, as a consequence, the noise in the AFM signal is also diminished.

Since the probe-liquid separation distance is sometimes hard to control precisely in experiments, high oscillation frequencies contribute to performing successful measurements by creating a margin of the probe lower position. In addition, more accurate NC-AFM data can be retrieved by increasing the probe frequency, even if the large $D_{\mathrm{e}}$ regime is not always attainable.

\section{Conclusions}

The dynamic response of a liquid film due to its interaction with an oscillating nano-probe was studied by means of numerical simulations. Our analysis yielded the wavenumber scales, that limit the range excited by the probe at each oscillation cycle and that corresponding to the natural film relaxation. The time scales which describe the transitory regime duration and the film relaxation were determined, as well as the phase duration along an oscillation cycle for the governing deformation mechanisms. The effects of the time-average probe position, the probe oscillation amplitude and frequency were analysed. For large separation distances, a theoretical solution in the wavenumber domain was obtained, which completely describes the dynamics of the film surface. Moreover, asymptotic behaviours for the probe oscillation frequency were derived from the solution. A quasi-static deformation regime is observed for low oscillation frequencies, whereas a frozen-like behaviour is found for high frequencies. For short separation distances, the solution provided evidence of critical combinations of oscillation parameters (time-average position, amplitude and frequency) that lead to prove wetting. Therefore, an educated selection of the oscillation parameters can be made based on our results, depending on the experimental objectives, instead of finding them heuristically.

It is important to notice that the present analysis does not include the effect of thermal agitation. Under this premise, our work should be considered as a first order approach, which yields the average behaviour of a liquid film interacting with an oscillating probe. A posteriori and extensive analysis, including higher order corrections (which should certainly scale with $k_{\mathrm{B}} T$ ), as it has been recently done for a static probe-bulk liquid interaction, ${ }^{35}$ must be taken into account in order to obtain more precise picture of the film interface.

The surface film dynamics, exposed in this paper, may be useful to understand liquid properties and their behaviour at the micro- and nanoscopic scales. Furthermore, it has been proven that the dynamic NC-AFM mode provides a non-intrusive tool to scan liquid samples, when a good choice of experimental parameters (frequency, free and set-point amplitude) is made. We expect that this work will lead to a quantitative understanding of the AFM imaging of soft samples, mainly in the recovery of unspoilt sample topographies. Additionally, the introduced ideas should also be applied to analyse AFM experimental results when a thin film of water covers the sample, as it is usually observed under uncontrolled humidity conditions. The importance of quantifying the effect of the adsorbed thin layer has been emphasised, ${ }^{15}$ because surface deformation and capillary effects may lead to false interpretations and to obtain inaccurate topographies.

\section{Appendix}

\section{(A) Hankel transform and inverse transform definitions}

The Hankel transform of order zero of a function $f$, defined in the spatial $r^{*}$ and temporal $t^{*}$ domains, is defined by:

$$
\begin{aligned}
g\left(k^{*}, t^{*}\right) & =\mathbb{H}_{0}\left\{f\left(r^{*}, t^{*}\right)\right\} \\
& =2 \pi \int_{0}^{\infty} f\left(r^{*}, t^{*}\right) J_{0}\left(k^{*} r^{*}\right) r^{*} \mathrm{~d} r^{*},
\end{aligned}
$$

whereas the inverse transform of a function $g$, defined in the angular wavenumber $k^{*}=R k$ and time $t^{*}=t / \tau$ domains, is given by:

$$
\begin{aligned}
f\left(r^{*}, t^{*}\right) & =\mathbb{H}_{0}^{-1}\left\{g\left(k^{*}, t^{*}\right)\right\} \\
& =\frac{1}{2 \pi} \int_{0}^{\infty} g\left(k^{*}, t^{*}\right) J_{0}\left(k^{*} r^{*}\right) k^{*} \mathrm{~d} k^{*},
\end{aligned}
$$

where $J_{0}$ is the zero-order Bessel function of the first kind.

\section{(B) Upper wavenumber cutoff}

At the first stages of the phenomenon, the upper cutoff $k_{\max } *$ corresponds to the wavenumber at which the growth rate is maximum. At $t^{*}=0$, the film free surface is flat $\eta^{*}=0$ and, as a consequence, its related wavenumber distribution is $\mathscr{N}^{*}=0$. Nevertheless, the growth rate $\partial \mathscr{N}^{*} / \partial t^{*}$ is not null at this stage. If one applies:

$$
\frac{\partial}{\partial k^{*}}\left[\frac{\partial \mathscr{N}^{*}}{\partial t^{*}}\right]=0 \text { with } \mathscr{N}^{*}=\frac{\partial \mathscr{N}^{*}}{\partial k^{*}}=0
$$


to eqn (18), and solves for $k^{*}$ at $t^{*}=0$, one finds the prime wavenumber $k^{*}=k_{\max }{ }^{*}$ that is instantaneously excited for a given separation distance $D^{*}$. This procedure leads, as a first step, to the expression:

$$
2 \mathscr{Q}^{*}+k^{*} \frac{\partial \mathscr{Q}^{*}}{\partial k^{*}}=0
$$

Making $\eta^{*}=0$ in eqn (12b) and applying the Hankel transform, the interaction potential $\mathscr{Q}^{*}$ can be simplified in the same way as for eqn (28). Taking into account this complexity reduction, and making $k^{*}=k_{\max }{ }^{*}$, the following non-linear implicit equation is obtained:

$$
k_{\max } * \sqrt{\left[D^{*}\right]^{2}-1}\left\{\frac{K_{1}\left(k_{\max } * \sqrt{\left[D^{*}\right]^{2}-1}\right)}{K_{2}\left(k_{\max } * \sqrt{\left[D^{*}\right]^{2}-1}\right)}\right\}=2,
$$

which relates the wavenumber $k_{\max }{ }^{*}$ and the separation distance $D^{*} . K_{1}$ and $K_{2}$ are first and second order modified Bessel function of the second kind. An expansion to the first order of the left hand-side term around $k_{\max }{ }^{*}=2 \pi$, the wavenumber naturally given by the probe radius, yields eqn (26). In Fig. 13, where $k_{\max }{ }^{*}$ is displayed as a function of $D^{*}$, good agreement between the exact solution of eqn (38) and the approximation given by eqn (26) is observed over the entire domain of $D^{*}$.

\section{(C) Wavenumber analytical solution}

Eqn (28) allows us to rewrite eqn (18), which becomes a linear non-homogeneous ODE with analytical solution. Considering a flat surface as the problem initial condition, i.e. $\eta^{*}=\mathscr{N}^{*}=0$ at $t^{*}=0$, the expression of $\mathscr{N}^{*}\left(q^{*}, t^{*}\right)$ is thus provided by:

$$
\mathscr{N}^{*}=\frac{\pi H_{\mathrm{a}}\left[k^{*}\right]^{4}}{4 \xi^{*}} \int_{0}^{t^{*}} \mathscr{Q}_{\mathrm{s}}^{*}\left(k^{*}, D^{*}\right) \exp \left(-\nu^{*}\left[t^{*}-r^{*}\right]\right) \mathrm{d} r^{*},
$$

where the probe position is temporarily given by $D^{*}=D^{*}\left(r^{*}\right)$, $r^{*}$ being a time integration variable. Moreover, as $\mathscr{Q}_{\mathrm{s}} *$ is an even function, it can be decomposed into a Fourier cosine series, which is represented for $D^{*}\left(t^{*}\right)$ by:

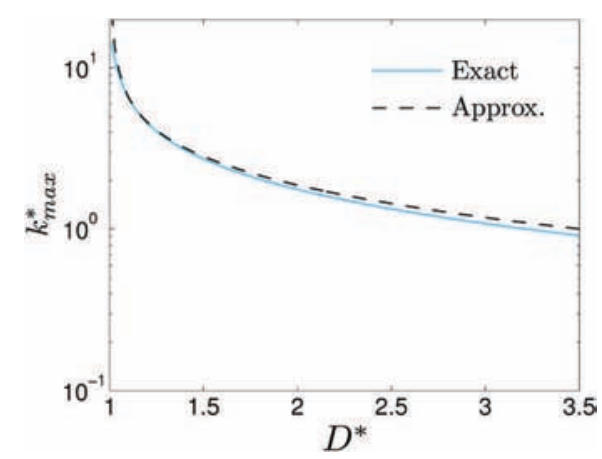

Fig. 13 Wavenumber $k_{\max }$ *, corresponding to the maximum growth rate of the wavenumber distribution at $t^{*}=0$. The exact solution of eqn (38) and the approximation obtained with eqn (26) are shown.

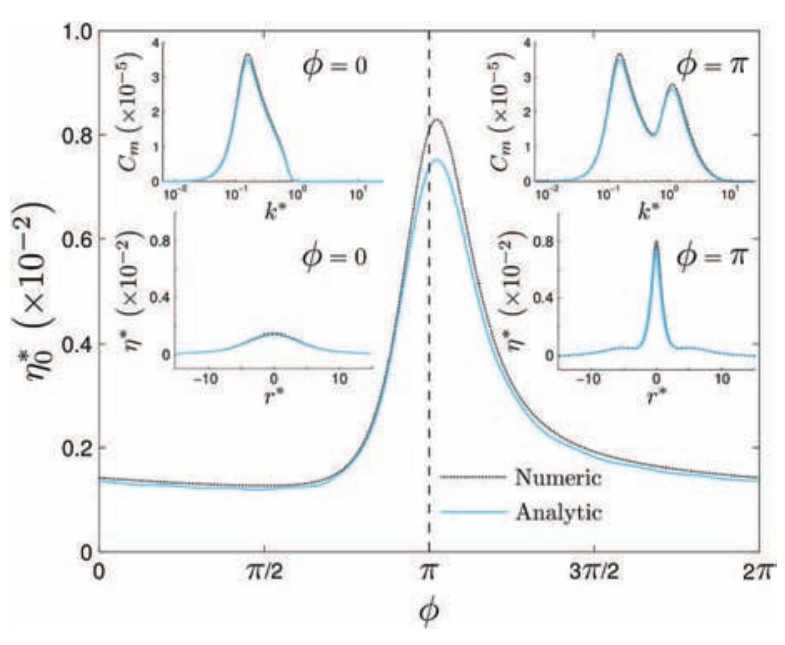

Fig. 14 Surface apex position $\eta_{0} *$ as a function of phase $\phi$, obtained from the numerical solution of eqn (18) and the analytical approximation given by eqn (29) with $j=10$ coefficients, and for $\overline{D^{*}}=2.2121$, $A^{*}=1, \omega^{*}=3 \times 10^{-1}$ and $p=199$. Inlets display the Fourier-Bessel coefficients $C_{m}$ as a function of the wavenumber $k^{*}$ and the surface position $\eta^{*}$ as a function of the radial position $r^{*}$, for $\phi=0$ and $\phi=\pi$.

$$
\begin{aligned}
\mathscr{Q}_{\mathrm{s}}{ }^{*}\left(k^{*}, D^{*}\right) & =\frac{\nu^{*} a_{0}}{\left[k^{*}\right]^{4}}+\sum_{j=1}^{\infty} \frac{a_{j} \sigma_{j}}{\left[k^{*}\right]^{4}} \cos \left(j \omega^{*} t^{*}\right), \\
a_{0}\left(k^{*}\right) & =\frac{\left[k^{*}\right]^{4} \omega^{*}}{2 \pi \nu^{*}} \int_{0}^{2 \pi / \omega^{*}} \mathscr{Q}_{\mathrm{s}}{ }^{*}\left(k^{*}, D^{*}\right) \mathrm{d} t^{*}, \\
a_{j}\left(k^{*}\right) & =\frac{\left[k^{*}\right]^{4} \omega^{*}}{\pi \sigma_{j}} \int_{0}^{2 \pi / \omega^{*}} \mathscr{Q}_{\mathrm{s}}{ }^{*}\left(k^{*}, D^{*}\right) \cos \left(j \omega^{*} t^{*}\right) \mathrm{d} t^{*},
\end{aligned}
$$

where $\sigma_{j}$ is given in eqn (30). An in-depth analysis indicates that $a_{0}$ depends only on the time-average probe position $\overline{D^{*}}$ and oscillation amplitude $A^{*}$, rather than on the angular frequency $\omega^{*}$. The Fourier decomposition allows us to find eqn (29). A comparison between the numerical solution and the approximation given by eqn (29), with $j=10$ coefficients, is shown in Fig. 14. Excellent agreement has been found, mainly in the qualitative behaviour of the film surface, in both space and wavenumber domains. The magnitude difference, obviously due to the approximation $D^{*} \gg \xi^{*} \eta^{*}$, is $10 \%$ at the most in space and less than $5 \%$ in the wavenumber domain.

\section{References}

1 T. Ondarcuhu and J. Aime, Nanoscale Liquid Interfaces: Wetting, Patterning and Force Microscopy at the Molecular Scale, Pan Stanford Publishing, 2013.

2 U. Landman, W. Luedtke, N. Burnham and R. Colton, Science, 1990, 248, 454-461.

3 H. Butt, B. Cappella and M. Kappl, Surf. Sci. Rep., 2005, 59, 1152.

4 J. Lenhard, Philos. Sci., 2006, 73, 605-616.

5 L. Kuipers and J. Frenken, Phys. Rev. Lett., 1993, 70, 39073910.

6 L. Kuipers, M. Hoogeman and J. Frenken, Surf. Sci., 1995, 340, 231-244. 
7 M. Forcada, M. Jakas and A. Gras-Marti, J. Chem. Phys., 1991, 95, 706-708.

8 N. Chen and B. Bhushan, J. Microsc., 2005, 221, 203-215.

9 R. Ledesma-Alonso, D. Legendre and P. Tordjeman, Phys. Rev. Lett., 2012, 108, 106104.

10 R. Ledesma-Alonso, P. Tordjeman and D. Legendre, Phys. Rev. E: Stat., Nonlinear, Soft Matter Phys., 2012, 85, 061602.

11 R. Ledesma-Alonso, D. Legendre and P. Tordjeman, Langmuir, 2013, 29, 7749-7757.

12 J. Tamayo and R. Garcia, Langmuir, 1996, 12, 4430-4435.

13 S. Herminghaus, A. Fery and D. Reim, Ultramicroscopy, 1997, 69, 211-217.

14 C. Mate and V. Novotny, J. Chem. Phys., 1991, 94, 8420-8427.

15 L. Zitzler, S. Herminghaus and F. Mugele, Phys. Rev. B: Condens. Matter Mater. Phys., 2002, 66, 155436.

16 T. Pompe, A. Fery and S. Herminghaus, Langmuir, 1998, 14, 2585-2588.

17 A. Fery, T. Pompe and S. Herminghaus, J. Adhes. Sci. Technol., 1999, 13, 1071-1083.

18 T. Pompe and S. Herminghaus, Phys. Rev. Lett., 2000, 85, 1930-1933.

19 F. Mugele, T. Becker, R. Nikopoulos, M. Kohonen and S. Herminghaus, J. Adhes. Sci. Technol., 2002, 16, 951-964.

20 A. Checco, P. Guenoun and J. Daillant, Phys. Rev. Lett., 2003, 91, 186101.

21 A. Checco, C. Yuguang, O. Gang and B. Ocko, Ultramicroscopy, 2006, 106, 703-708.
22 A. Checco, H. Schollmeyer, J. Daillant and P. Guenoun, Langmuir, 2006, 22, 116-126.

23 A. Checco, O. Gang and B. Ocko, Phys. Rev. Lett., 2006, 96, 056104.

24 J. Aime, D. Michel, R. Boisgard and L. Nony, Phys. Rev. B: Condens. Matter Mater. Phys., 1999, 59, 2407-2416.

25 R. Boisgard, J. Aime and G. Couturier, Surf. Sci., 2002, 511, 171-182.

26 L. D. Landau and E. M. Lifshitz, Fluid Mechanics, Oxford, Pergamon Press, 1987.

27 T. Salez, J. McGraw, O. Bumchen, K. Dalnoki-Veress and E. Raphal, Phys. Fluids, 2012, 24, 102111.

28 A. Oron, S. Davis and S. Bankoff, Rev. Mod. Phys., 1997, 69, 931-980.

29 H. C. Hamaker, Physica, 1937, 4, 1058-1072.

30 P.-G. de Gennes, F. Brochard-Wyart and D. Quere, Capillarity and Wetting Phenomena: Drops, Bubbles, Pearls, Waves, Springer, 2003.

31 J. Berthier and P. Silberzan, Microfluidics for Biotechnology, Artech House, 2nd edn, 2009.

32 J. Berthier and K. Brakke, The Physics of Microdroplets, WileyScrivener, 2012.

33 D. Quinn, J. Feng and H. Stone, Langmuir, 2013, 29, 14271434.

34 M. Guizar-Sicairos and J. Gutierrez-Vega, J. Opt. Soc. Am. A, 2004, 21, 53-58.

35 T. Bickel, Europhys. Lett., 2014, 106, 5. 